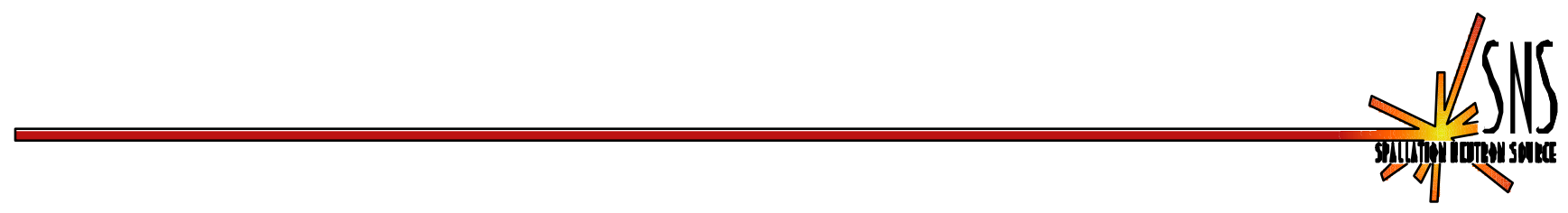

\title{
Mechanism of Electron Multipacting With Long-Bunch Proton Beam
}

BNL/SNS Technical Note

No. 132

L. Wang, M. Blaskiewicz, J. Wei, BNL, Upton, $N Y$, USA

K. Ohmi, KEK, Tuskuba, Japan

R. Macek, LANL, Los Alamos, NM, USA

January 16, 2004

Collider-Accelerator Department

Brookhaven National Laboratory

Upton, New York \#11973 


\title{
MECHANISM OF ELECTRON MULTIPACTING WITH A LONG-BUNCH PROTON BEAM
}

\author{
L. Wang, M. Blaskiewicz, J. Wei, BNL, Upton, NY, USA \\ K. Ohmi, KEK, Tuskuba, Japan \\ R. Macek, LANL, Los Alamos, NM, USA
}

\begin{abstract}
The mechanism of electron multipacting in long bunched proton machine has been quantitatively described by the electron energy gain and electron motion. Some important parameters related to electron multipacting are investigated in detail. It is proved that multipacting is sensitive to beam intensity, longitudinal beam profile shape and transverse beam size. Agreement is achieved among our analysis, simulation and experiment.
\end{abstract}

PACS numbers: 29.27.Bd, 29.20.Lq

\section{INTRODUCTION}

The problem of transverse instability and beam loss due to electron-proton interaction has persisted over many years. It was first observed at INP PSR in 1965[1]. Shortly thereafter, electron cloud and beamintroduced multipacting was found at CERN-ISR [2, 3]; this instability was detected during the coasting beam operation and was cured using clearing electrodes. More recently, transverse instability was reported in a bunched proton beam in a proton synchrotron ring at Los Alamos National Laboratory (LANL-PSR) [4]. It appeared to be caused by an electron cloud. An instability, which seems to be due to the interaction of electrons and protons, also occurred at the AGS booster [5]. Electron-cloud instability has been found since the 1990s in the PF [6, 7], KEKB [8], PEP-II [9], BEPC [10], PS, and SPS [11-12]. Grobner suggested that beam-induced multipacting causes an electron cloud to accumulate inside the vacuum chamber $[13,14]$. The cloud then interacts with the proton- or positron-beam and hence, destabilizes it. Experimental observations of electron-cloud instabilities differ distinctively for "short bunches" where multibunch multipacting is expected to be important (the PS, SPS, and B factories) and "long bunches" where single-bunch, trailing-edge multipacting probably is dominant. The mechanism of beam-induced multipacting seems quite different for the two.

In this article, we discuss electron cloud build-up in a long proton machine. The PSR is the existing proton machine in which strong electron-cloud instability was reported. Two candidate mechanisms were offered to qualitatively explain this observation $[4,15]$. In the first, electrons captured by the beam (e.g., from residual gas ionization or electrons that survive the gap) oscillate in the potential well of the proton 
beam, emerging at the end of the pulse with energies that depend on initial conditions and the beam's intensity. When these electrons strike the wall, secondary electrons are produced with yields exceeding unity. The secondaries may travel to the opposite wall and reflect or make tertiary electrons. Such interactions with the wall degrade the electrons' energies to a few eVs; in these cases, it can take many nanoseconds for them to die out. If a large enough fraction survives the gap, they will accumulate or buildup until the production and loss rates are in equilibrium. The second candidate mechanism is based on what is aptly described as "trailing edge multipactor". Electrons born at the wall near or after the peak of the pulse passes will be accelerated towards the center of the beam and decelerated after passing through it. On the trailing edge of the beam pulse, such electrons will reach the opposite wall with a certain energy gain. If the gain is high enough, then the secondary emission yield (SEY) can exceed unity, resulting in amplification on each successive traversal of the beam pipe.

Many studies have been made of electron-cloud buildup in long proton machines but all are based on numerical methods [16-20]. The buildup depends on many factors, such as the shape of the beam's longitudinal profile, bunch current, chamber size, and the SEY. Even if some factor is found to be a key one in simulation studies, it still is unclear that this is the case from the physics point of view. For example, Pivi and Furman found that the shape of the longitudinal beam profile is important [17]. By artificially cutting the beam's tail, they demonstrated a rapid reduction in electron density. However, this phenomenon has not yet been explained. The very important concept of the trailing edge multipactor is only explained qualitatively so far $[4,15]$ as described above. The gain in electron energy is estimated using simple formulae based on linear approximations [16, 19]. These estimations are helpful. However, considering that energy gain is the most vital factor in electron multipacting, we developed a more realistic estimation, and in this paper we apply it to the analysis of multipacting. It is still problematic why electron clouds are important in some accelerator machines, such as the PSR, but not in other machines, such as the ISIS. This topic was widely discussed in the electron cloud workshop, ECLOUD02. Many numerical studies tried to find the difference in the electron cloud in different machines by simulating their electron density $[17,18$ and 20]. But, because many factors are related electron build-up, such kinds of simulation fail to give a clear physics explanation of the root causes of these differences. For example, Ohmi and his colleagues used a half-sinuous beam profile in their simulation [18]; however, Furman and Pivi assumed that the beam's profile was parabolic [20]. Different beam profiles may generate very different results, as we prove in this paper.

The objective of our research, reported here, was to quantify the mechanism of multipacting in the long proton beam. We confirmed our analysis with the simulation code CLOUDLAND [21]. Our results give us a clearer physics basis to explain the electron cloud build-up in long bunch machine. This knowledge is very helpful, and may answer some of the questions raised in the previous paragraph that the numerical methods could not resolve. Furthermore, we investigated several important factors related to multipacting one by one, instead of considering them together. Therefore, we can clearly delineate the effect of each one.

This paper is organized as follows. 
First, we introduce the SEY and physics model used in the code CLOUDLAND.

Second, we analyze electron motion under the beam's space-charge force and dipole magnetic field. Long bunches can deeply trap electrons which then are emitted or exist before the bunch center. On the other hand, electrons emitted after the center of a proton bunch will drift straight to the opposite wall's surface. Accordingly, there is no loss of electrons before the bunch's center, which can partly explain why multipacting always occurs at the bunch's tail. The gain in electron energy is analyzed as a function of the beam's longitudinal and transverse profile, its transverse size, and the chamber's size. One very important factor, the longitudinal beam profile factor, is defined. According to this factor, the gain in electron energy is usually bigger at the bunch's tail, which can explain the mechanism of so-called "trailing edge multipactor".

Third, a few significant parameters related to electron multipacting were investigated in greater detail, based on analyses, simulations, and experimental results. Among of them, beam intensity, longitudinal beam profile shape, transverse beam size, beam in gap, peak SEY and energy at peak SEY are demonstrated to be important in multipacting and beam instabilities. Beam instabilities are sensitive to electrons by ionization but multipacting is not.

\section{SEY AND THE PHYSICS MODEL}

ORNL is constructing a Spallation Neutron Source (SNS), equipped with a high intensity proton synchrotron. As examples, we use the SNS ring and PSR beam in this study. Table 1 shows the beams' parameters. The SNS beam is assumed to be cylindrical with uniform distribution in the transverse plane, and the PSR beam a Gaussian one.

Table 1 Simulation parameters for the SNS and PSR

\begin{tabular}{cccc}
\hline \hline Parameter & Description & SNS & PSR \\
$\mathrm{E}(\mathrm{GeV})$ & Beam energy & 1.9 & 1.75 \\
$\mathrm{C}(\mathrm{m})$ & Circumference & 248 & 90 \\
$\mathrm{~N}_{\mathrm{p}}$ & Beam intensity & $2.05 \times 10^{14}$ & $5 \times 10^{13}$ \\
$\mathrm{a}_{\mathrm{x}}, \mathrm{a}_{\mathrm{y}} / \sigma_{\mathrm{x}}, \sigma_{\mathrm{y}}(\mathrm{mm})$ & Transverse beam size & 28,28 & 10,10 \\
$\tau_{\mathrm{b}}(\mathrm{ns})$ & Bunch length & 700 & 250 \\
$\mathrm{~b}(\mathrm{~cm})$ & Beam pipe radius & 10 & 5 \\
$P_{l}$ & Proton loss rate/per turn & $1.1 \times 10^{-6}$ & $4.0 \times 10^{-6}$ \\
$\mathrm{Y}$ & Proton-electron yield & 100 & 100 \\
\hline \hline
\end{tabular}

A major unknown factor is the number of electrons born at the wall. In the PSR, this number at any given location is uncertain by at least two orders-of-magnitude. It is difficult to reliably estimate the electron yield from proton losses. We need to know the grazing angle of incidence very well for the lost protons, and the places where they are lost. We do not have experimental results. Conceptually, the number of initial electrons born at the wall might be treated as being proportional to the instantaneous line density of protons in the region of interest (assuming the losses are proportional to line density) with the proportionality constant a free parameter to be fixed by comparing the simulations to one set of experimental data. In Table I, a uniform rate of proton loss along the ring (which is far from true in the real machine), and a proton- 
electron yield of 100 were assumed based on comparing the simulation and experimental data from the PSR. When these proton-generated electrons hit the beam chamber's surface after a period of drifting, more electrons, called secondary electrons, are produced. The emission of secondary electrons is an important process for the buildup of the electron cloud. The secondary electrons include three types: backscattered electrons, rediffused electrons, and true secondary electrons [22]. Secondary emission yield is defined as a fraction of the number of electrons emitted from the metal surface to the total number of incident electrons. In cases where the SEY is larger than unity, the number of electrons increases exponentially. This phenomenon is called multipacting. The yield of backscattered electrons with normal incident angle is

$$
\delta_{e}\left(E_{0}\right)=P_{1, e}(\infty)+\left[\hat{P}_{1, e}-P_{1, e}(\infty)\right] e^{-\left(\mid E_{0}-\hat{E}_{e} / / W\right)^{p} / p} .
$$

where $E_{0}$ is the energy of the incident electrons. The yield of rediffused electrons with normal incident angle is

$$
\delta_{r}\left(E_{0}\right)=P_{1, r}(\infty)\left[1-e^{-\left(E_{0} / E_{r}\right)^{r}}\right] .
$$

The yield of the true secondary electrons with normal incident angle is

$$
\delta_{t s}\left(E_{0}\right)=\frac{s\left(E_{0} / \hat{E}_{t s}\right) \hat{\delta}_{t s}}{s-1+\left(E_{0} / \hat{E}_{t s}\right)^{s}} .
$$

The variables in Eqs. (1-3), except $E_{0}$, are a constant number that depends on the material properties of the chamber's surface.

Figure 1 shows the SEY used for simulation and Table 2 the secondary emission parameters. The true secondary parameters are based on one of the experimental results in CERN. Cimino recently showed that the yield of reflected electrons with zero energy, $\hat{P}_{1, e}$ in Table 2 , could be close to 1 [23]. Therefore, the reflected component plays a major role in $\delta$ at low energies. The multipacting strongly depends on the SEY parameters. We focus on the physics of multipacting in this study. In a real storage ring, the measured SEY parameters should be applied in estimating electron multipacting

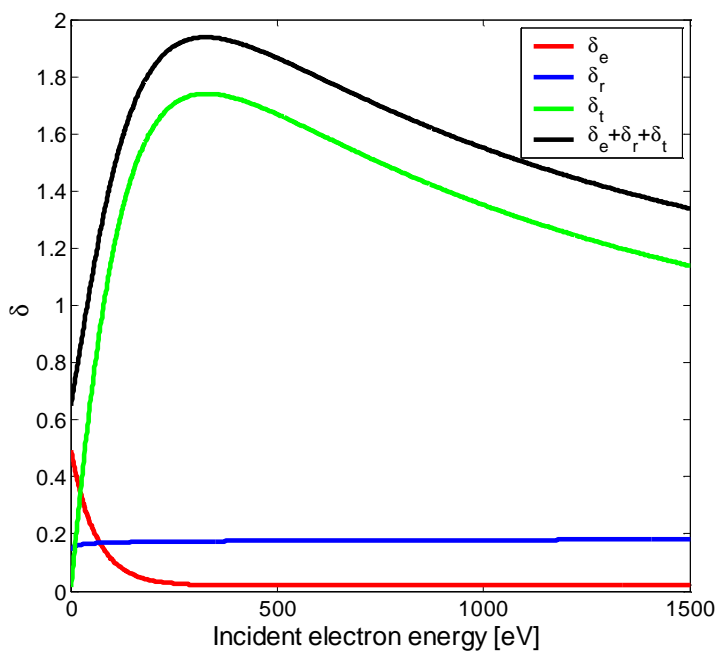

Figure 1 Secondary emission yield
Table 2 Main parameters of SEY

\begin{tabular}{cc}
\hline \hline Backscattered electrons & 0.02 \\
$P_{1, e}(\infty)$ & 0.5 \\
$\hat{P}_{1, e}$ & 0 \\
$\hat{E}_{e}(\mathrm{eV})$ & 60 \\
$W(\mathrm{eV})$ & 1 \\
$P$ & 0.19 \\
Rediffused electrons & 0.041 \\
$P_{1, r}(\infty)$ & 0.104 \\
$\hat{E}_{r}(\mathrm{eV})$ & 330 \\
$R$ & 1.74 \\
True secondary electrons & 1.52553 \\
$\hat{E}_{t s}(\mathrm{eV})$ & \\
$\hat{\delta}_{t s}$ & \\
$\mathrm{~S}$ &
\end{tabular}


The simulation program we used is a three-dimensional particle-in-cell (PIC) code named CLOUDLAND [21]. It includes the three-dimension electron-space charge, beam-electron interaction, and various magnetic fields and electric fields. A primary electron is emitted when a lost proton hits the wall. The electrons move under the beam and its space charge. Inside magnets, the magnetic field also should be included in the calculations. When an electron hits the vacuum chamber's surface, it generates secondary electrons. A statistic distribution generator obeying the experimental results controls the SEY, energy, and emission angle. Similarly, the secondary electrons may generate tertiary electrons. Because the SEY strongly depends on the energy of the incident electrons, multipacting has very closer relation with electron motion.

\section{PARTICLE MOTION}

The primary electrons are produced by beam loss at the chamber's surface and ionization at the beam's position. If an electron can oscillate many times under the bam force during the passage of one bunch, then the bunch is called long bunch. Assuming the bunch length is $2 \hat{z}$ and the average frequency of electron oscillation is $\bar{\varpi}$, a long bunch should satisfy

$$
\frac{\hat{z} \bar{\omega}}{\pi \beta c} \gg>1
$$

where $\beta$ is the velocity of the proton normalized by the speed of light, $c$. Both the SNS and PSR beam are long beams, wherein the trapped electrons can oscillate more than 50 periods during the bunch's passage.

\section{Magnetic Field Free Region}

In the field-free region, the electrons move under the space-charge fields of the proton beam and between other electrons. The space-charge field of the electron cloud can be neglected during the beam's passage because the neutralization factor is small except at the bunch tail where strong multipacting usually happens. Therefore, the electrons' motion during beam passage can be approximated as a movement under the beam's space-charge field only. For the long proton bunch, the longitudinal space-charge field due to potential variations in longitudinal direction also can be neglected because of the slow variation in, and the symmetry of, the longitudinal beam profile that traps the particles in a longitudinal direction. Therefore, the electrons mainly move under the transverse beam fields.

The SNS's transverse beam profile is close to a square with a uniform distribution resulting from correlated painting during injection. Including the space charge causes rapid diffusion in the azimuthal direction and results in round shape [24]. A cylindrical transverse profile is assumed in this paper to approximate the real distribution of the SNS beam.

For a cylindrical beam with a uniform distribution in transverse section, the space-charge fields are 


$$
E_{r}(t)=\left\{\begin{array}{ll}
\frac{\lambda(t)}{4 \pi \varepsilon_{0}} \frac{2}{r} & (\mathrm{r}>a) \\
\frac{\lambda(t)}{4 \pi \varepsilon_{0}} \frac{2 r}{a^{2}} & (\mathrm{r}<a)
\end{array} .\right.
$$

where $\mu_{0}=4 \pi \times 10^{-7} \mathrm{H} / \mathrm{m}$ is called the permeability of vacuum, $\varepsilon_{0}=10^{-9} / 36 \pi \mathrm{F} / \mathrm{m}$ is known as the permittivity of vacuum, $\lambda$ is the beam line density, and $a$ is the transverse beams' size. The electron oscillates slowly in longitudinal direction (beam's direction) with small amplitude and rotates in the azimuth direction with constant angle velocity that depends on the initial condition. Since the motion in the radial direction is uncoupled with the other direction, the nonlinear Hamiltonian of the radial motion is obtained as

$$
H=\frac{p^{2}}{2 m}+e U(r, t)
$$

with

$$
U(r, t)=\left\{\begin{array}{ll}
\frac{\lambda(t)}{4 \pi \varepsilon_{0}}\left(1+2 \ln \frac{r}{a}\right) & (\mathrm{r}>a) \\
\frac{\lambda(t)}{4 \pi \varepsilon_{0}} \frac{r^{2}}{a^{2}} & (\mathrm{r}<a)
\end{array} .\right.
$$

The electron motion in radial direction is a "nearly periodic oscillation" and has a slow time-dependence given by function $\lambda(t)$. Assuming constant $\lambda$, the electron will make an exact periodically nonlinear oscillation. In the maximum oscillation amplitude $r_{a m p}$, the kinetic energy is zero. To get the period of nonlinear oscillations, this is integrated over one-fourth oscillation period

$$
T=4.0 \int_{0}^{r_{a n p}} \frac{d r}{v(r)}=4.0 \int_{0}^{r_{a m p}} \frac{d r}{\sqrt{2 \Phi e / m}}
$$

with

$$
\Phi(r)= \begin{cases}\frac{\lambda}{2 \pi \varepsilon_{0}} \ln \frac{r_{a m p}}{r} & \left(\mathrm{r}>a ; r_{a m p}>a\right) \\ \frac{\lambda}{4 \pi \varepsilon_{0}}\left(1+2 \ln \frac{r_{a m p}}{a}-\frac{r^{2}}{a^{2}}\right) & \left(\mathrm{r} \leq a ; r_{a m p}>a\right) \\ \frac{\lambda}{4 \pi \varepsilon_{0} a^{2}}\left(r_{a m p}^{2}-r^{2}\right) & \left(\mathrm{r} \leq r_{a m p} \leq a\right)\end{cases}
$$

where $m$ is the mass of the electron. Substituting $\Phi$ of Eq. (9) for that of Eq.(8), we get the period of nonlinear motion as 


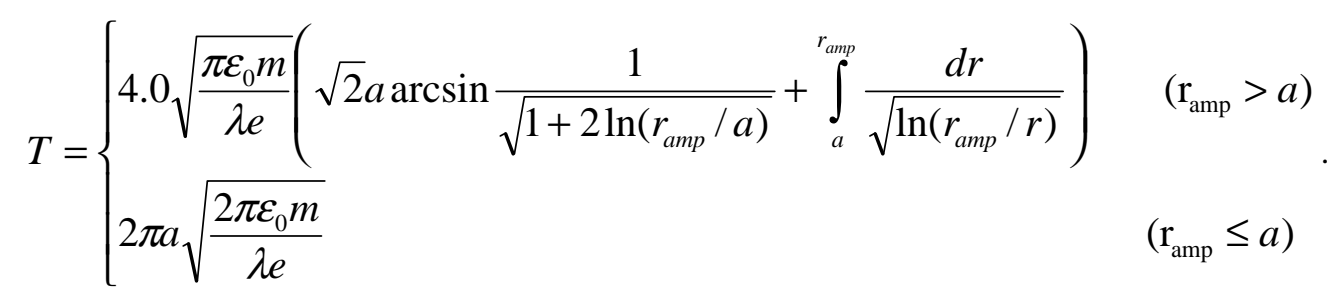

The electrons by ionization are produced at the beam with radial coordinates smaller than the beam's size. Therefore, these electrons will oscillate under the linear force. However, the electrons produced by beam loss at the beam pipe will oscillate under the nonlinear force. Consequently, oscillator frequency depends on the radial coordinate when $\mathrm{r}_{a m p}>a$ due to the effect of the nonlinear force.

If the beam line density $\lambda(t)$ does not change very much within one period of electron oscillation,

$$
\frac{1}{\omega_{e}^{2}} \frac{d \omega_{e}}{d t}<<1
$$

there is an adiabatic invariant which is defined with canonical variables $p$ and $q$ as

$$
J=\oint p d q .
$$

For the SNS beam, condition (11) is satisfied except during the first and last 20ns of the bunch's pulse. The total beam pulse is $700 \mathrm{~ns}$. Therefore, the adiabatic invariant exists during most of the beam passage.

For a given oscillation amplitude $r_{a m p}, p(r)$ can be written as

$$
p(r, t)=\sqrt{2 \operatorname{em}\left(U\left(r_{\text {amp }}, t\right)-U(r, t)\right)}
$$

Substituting Eq. (7) and (13) into Eq.(12), we can get the motion invariant

$$
J= \begin{cases}\frac{\pi r_{a m p}^{2}}{a} \sqrt{\frac{m e \lambda}{2 \pi \varepsilon_{0}}} & \left(r_{a m p}<a\right) \\ 4 a \sqrt{\frac{m e \lambda}{2 \pi \varepsilon_{0}}}\left(\frac{\sqrt{2}}{2} x^{1 / 2}+\frac{1+2 x}{2} \operatorname{arctg} \frac{1}{\sqrt{2 x}}+\frac{\sqrt{2}}{a} \int_{a}^{r_{a m p}} \sqrt{\ln \frac{r_{a m p}}{r}} d r\right) \quad\left(r_{a m p}>a\right)\end{cases}
$$

where $x=\ln \left(r_{\text {amp }} / a\right)$

For a "smooth" longitudinal beam profile (continuous with its derivative), the variation in electron oscillation amplitude due to the variation of beam density's during the bunch passage can be calculated according to Eq. (14). Figure 2 gives an example of the amplitude calculated by Eq. (14) and of frequency by Eq. (10) during the beam's passage. The estimated amplitude, shown as the bold solid black line in the figure, agrees well with the numerically simulated oscillation amplitude of the particle. The oscillation frequency, which depends on the amplitude and beam's density, ranges from 20 to $140 \mathrm{MHz}$.

The oscillation frequency of electrons varies during the passage of the beam. Consequently, the proton beam oscillates coherently at a frequency range different from the above incoherent oscillation frequency by a factor that depends on the neutralization caused by coupling between electrons and the proton beam. Therefore, electron-proton instabilities can be distinguished from the conventional impedance-caused 
instability with a width of resonant frequency that depends on the beam. The peak beam spectrum is roughly proportional to $\sqrt{N_{p}}$ [25-26], and close to the incoherent frequency given by Eq. (10) because the neutralization factor is small. Therefore, Eq. (10) can be used to estimate the instability spectrum.

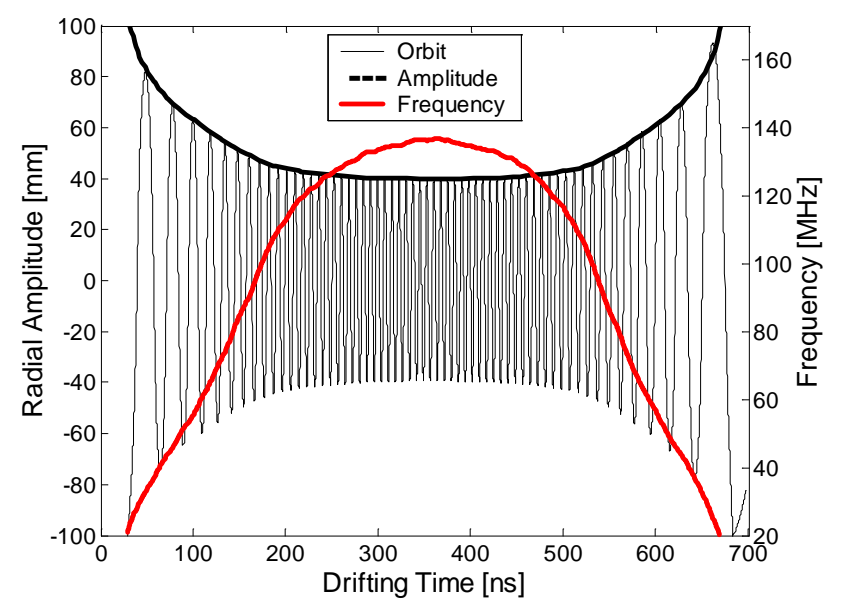

Figure 2 Oscillation amplitude and frequency. The bold solid black line is the estimated amplitude.

Figure 3 shows the amplitude contour distribution for the SNS beam profile, and Figure 4 shows the typical orbit obtained by the CLOULDLAND program; they are consistent. From these two figures, we concluded that

(1) All electrons remaining inside the chamber before the approaching bunch (electrons surviving from the last bunch gap) can be trapped inside the beam during the bunch's passage and be released at its end. The examples of such kinds of electron motion are depicted in Figure 4 with blue and red lines. The blue line shows that electrons surviving from the last bunch gap with oscillation amplitudes about the chamber's radius still can be trapped inside the beam. These surviving electrons from the last bunch gap are important for beam dynamics and cause beam instability because they can be deeply trapped inside beam and their number is huge. They have weak effect on multipacting due to their long term trapping and low energy at the chamber's surface.

(2) The electrons emitted at the pipe's surface between the bunch head and bunch center will oscillate during the beam's passage and hit the chamber wall after the bunch center at the moment

$$
t_{\text {hit }} \approx t_{\text {emission }}+2\left(t_{\text {bunchcenter }}-t_{\text {emission }}\right)
$$

due to the symmetry of the beam's profile. The earlier the electron is emitted, the later it hits the wall. Electrons emitted at the bunch head could be deeply trapped inside the beam. But more than 95\% of primary electrons oscillate with amplitude bigger than the beam's size. The black line depicts such an example of an electron's orbit (Figure 4). It is more clearly shown in Figure 3.

(3) The electrons produced at the beam by ionization can be trapped inside it until the whole bunch passes them. The pink line in Figure 4 gives the orbit of an electron produced by ionization. These electrons have similar effect as the electrons surviving from the last bunch gap. 
(4) The electrons emitted at the chamber's surface after the bunch center will drift straight to the opposite chamber wall and produce secondary electrons because there the beam's profile has a negative derivation. The secondary electrons continue cross the chamber until they hit the opposite chamber surface to generate tertiary electrons. As a result, the electrons generated after the bunch center are important for multipacting due to their having a short crossing time and sufficient energy when they hit the chamber surface at the bunch tail, as we discuss below. The green line in Figure 4 plots the orbit of a straight drifting electron. We call this kind of electron a straight drifting electron in this paper because its orbit is a straight line; all other electrons as described in (1-3) are termed trapped electrons. Figure 5 shows the three-dimensional orbit and phase plot of a trapped electron.

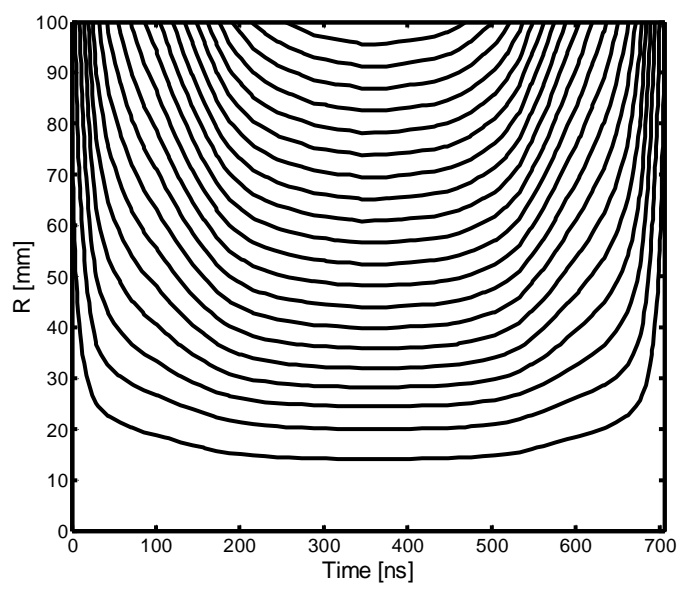

Figure 3 Contour plot of the oscillation amplitude resulting from adiabatic invariant for the SNS beam

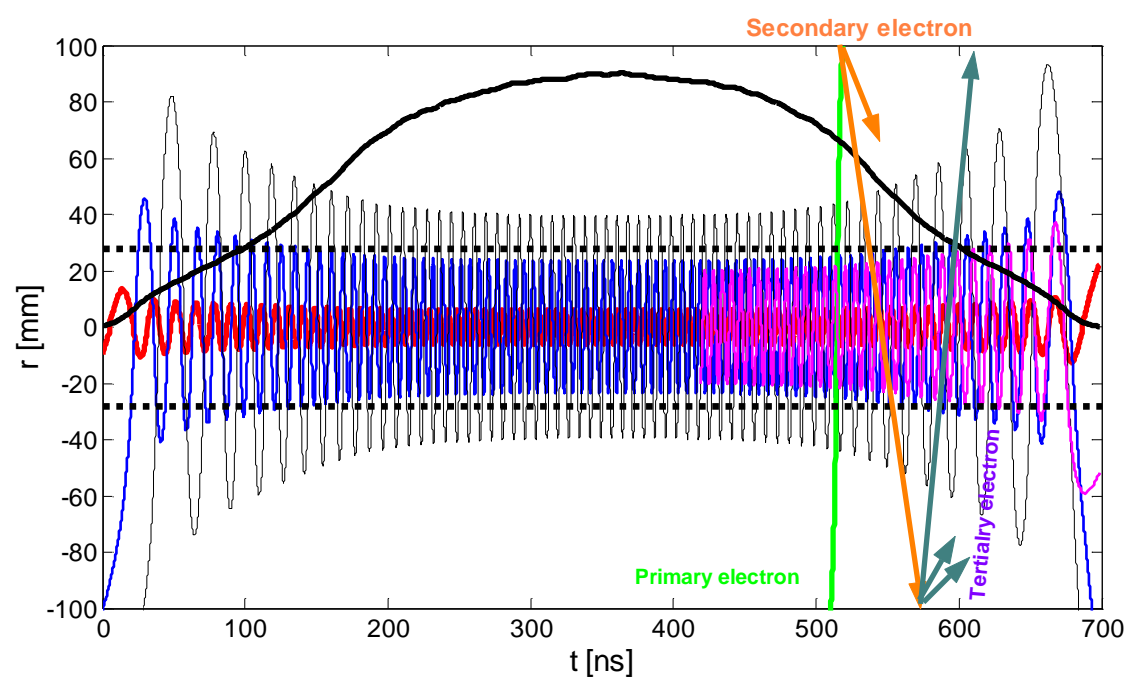

Figure 4 Typical orbits of various electrons trapped by the SNS beam; the bold solid line shows the shape of the longitudinal beam profile and the dashed black lines show its transverse size. The blue and red lines show the orbits of surviving electrons from the last bunch gap. They are trapped inside beam during the beam passage and can cause beam instabilities. The solid back line shows the orbit of the electron which is emitted at the chamber surface between bunch head and bunch center. It oscillates with large amplitude and 
lost between bunch center and tail. The green line shows the electron which is emitted at the chamber surface between bunch center and tail. It is important for multipacting. It generates secondary and tertiary electrons. The pink line shows the orbit of an electron generated by ionization.
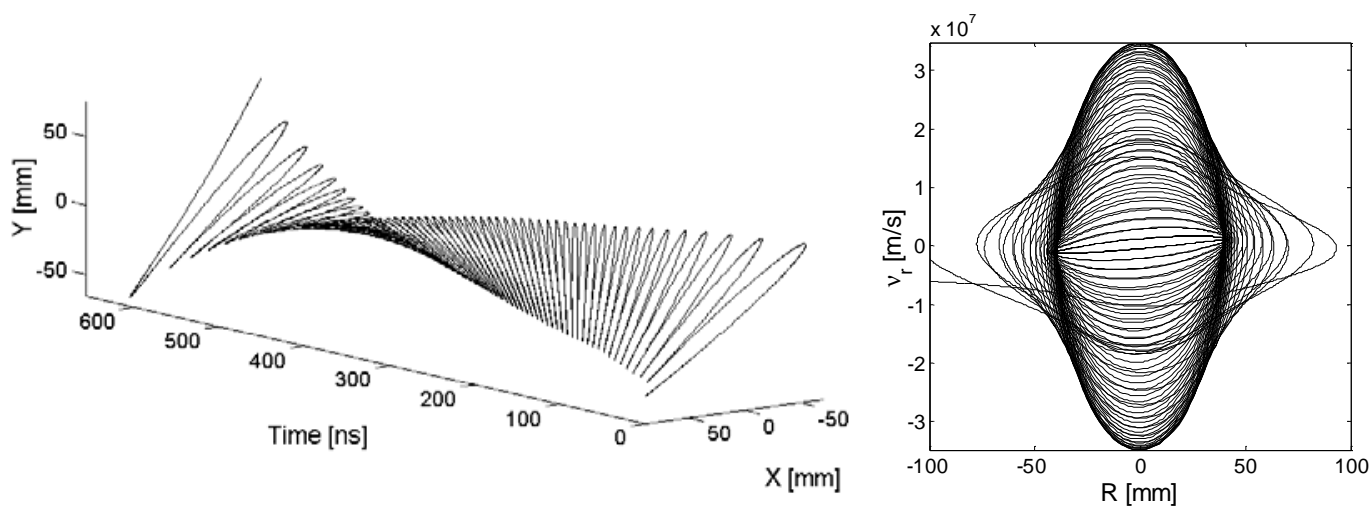

Figure 5 Three-dimensional orbit (left) and phase plot (right) of a trapped electron

If the transverse beam distribution is round Gaussian, the potential of the beam corresponding to Eq. (7) becomes

$$
U(r)=\frac{\lambda}{4 \pi \varepsilon_{0}} \int_{0}^{r} \frac{2}{t}\left[1-\exp \left(-\frac{t^{2}}{2 \sigma^{2}}\right)\right] d t .
$$

Similarly, we obtain the relationship of the electron oscillation amplitudes as in Eq. (12). The PSR beam has a Gaussian distribution in the transverse plane. Figure 6 shows the PSR beam's profile and the oscillation amplitude contour. The contour plots for the SNS and PSR are similar. Electrons can only hit the chamber wall surface after the bunch center. Therefore, multipacting can only occur after the bunch center.

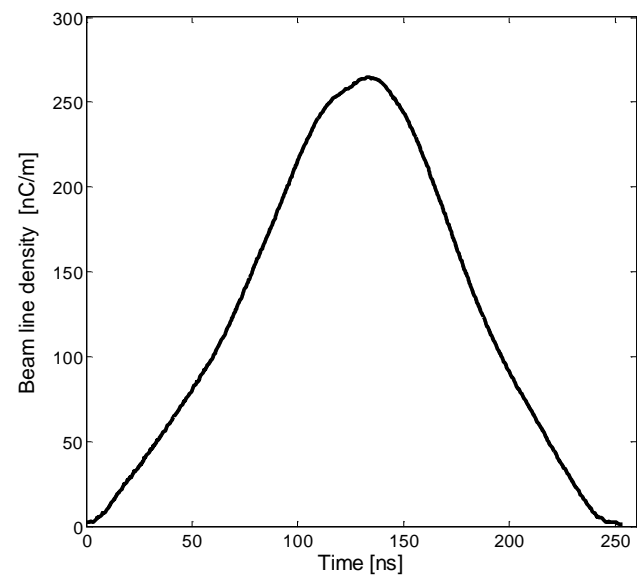

(a)

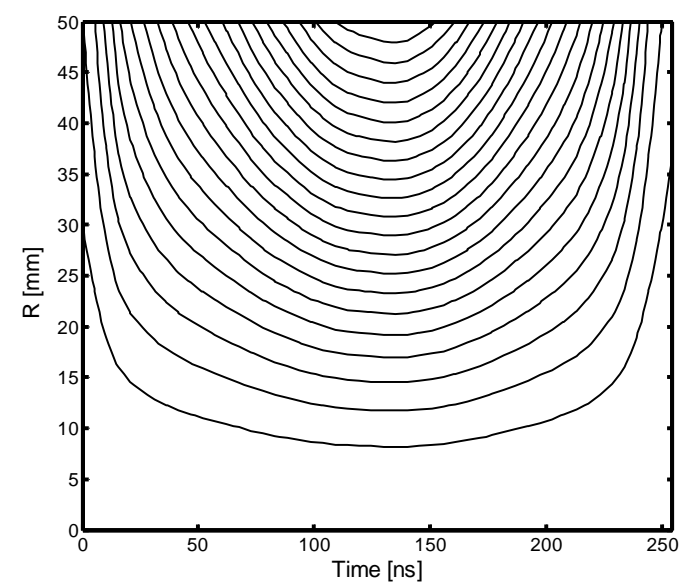

(b)

Figure 6 PSR beam profile (a) and electron oscillation amplitude contour (b)

The yield of primary-electron emission depends on the rate of beam loss and vacuum pressure. In a real machine, the yield of electrons by beam loss is at least one order-of-magnitude larger than that by ionization. Further, multipacting due to the electrons generated by ionization is very weak for a long bunch as discussed in this paper. As a result, the number of electrons formed by ionization can be ignored 
compared with those due to beam loss. The electrons by ionization may cause beam instabilities when the vacuum is poor. However, the multipacting due to these electrons is always weak for long beam as discussed in the next section. Therefore, we focus only on the electrons due to beam loss in this paper.

As Figures 3 and 4 show, all electrons existing before the bunch center will be trapped by the long beam and released after the bunch center, as Eq. (14) describes. The energy of these trapped electrons when they hit the beam chamber's wall depends on the initial conditions. We note that the electrons' motion is threedimensional. However, simulation shows that the maximum energy of these electrons is the same as that of the straight drifting electrons (Figure 7). The energy shown in this figure represents the energy of one electron when it hits the wall. The emission- and loss-time in the figure signify, respectively, the moment when one electron is emitted from the wall surface, or when one hits the wall's surface. The difference between these times gives the lifetime of an electron. The relationship of emission time and loss time roughly satisfies Eq. (15). There is no electron loss before the $350 \mathrm{~ns}$, the half-proton bunch length, as predicted. Most trapped electrons have a lower energy gain than the straight drifting electrons. Therefore, we can estimate the energy of straight drifting electrons to reach an understanding of the energy gain of all electrons. For SNS beam, an electron can hit the chamber surface about 30 times during the period from bunch center to bunch tail. It takes $10 \mathrm{~ns}$ to strike the surface once on average. On the other hand, the trapped electron takes long time to hit the surface once (Eq.15). Therefore, only straight drifting electrons are important for multipacting due to their short crossing time.

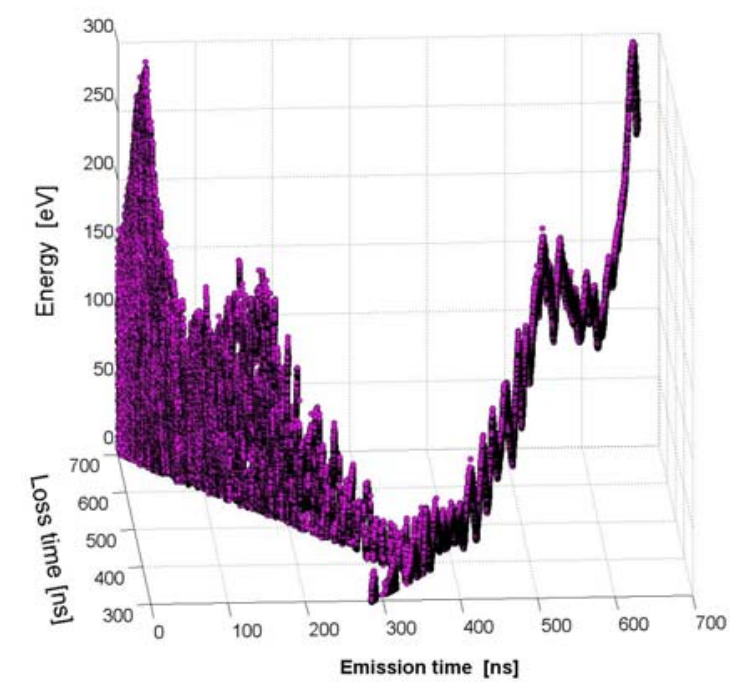

Figure 7 Relationship of energy gain, emission time, and loss time of the primary electrons in the SNS's drift region

One straight drifting electron emitted from the chamber surface will drift straight to the opposite wall's surface. The potential given by Eq. (7) varies during the beam's passage due to the variation of its line density. For convenience, we assume the following linear dependence

$$
U(r, t)=\lambda(t) u(r) .
$$


Figure 8 schematically plots the motion of an electron for a short interval. One electron moves from point $P_{1}$ to $P_{2}$ with beam line density $\lambda_{1}$, and then moves to $P_{3}$ with beam line density $\lambda_{2}$. During the movement from $P_{1}$ to $P_{3}$, the change of electron kinetic energy is

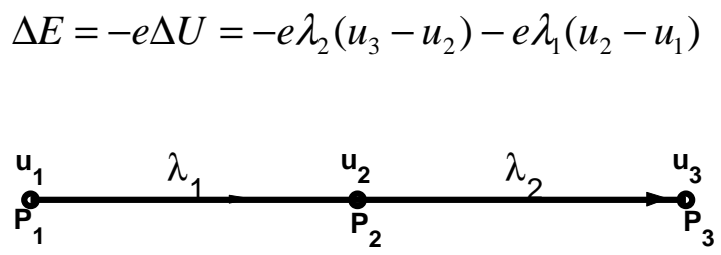

Figure 8 Scheme of electron energy gain

Rewriting Eq. (18), we get

$$
\Delta E=e\left(\lambda_{1} u_{1}-\lambda_{2} u_{3}\right)+e u_{2}\left(\lambda_{2}-\lambda_{1}\right)=e\left(\lambda_{1} u_{1}-\lambda_{2} u_{3}\right)+e u_{2} \Delta \lambda=\Delta E_{1}+\Delta E_{2}
$$

The first part of energy gain when the electron hits the wall is

$$
\Delta E_{1}=-e u(b) \Delta \lambda=-\frac{e \Delta \lambda}{4 \pi \varepsilon_{0}}\left(1+2 \ln \frac{b}{a}\right)
$$

where $b$ is the radius of the vacuum chamber and $\Delta \lambda$ is the difference in bunch density between the moments of emission and of loss . Note that $\Delta \lambda<0$ for straight drifting electron. Assuming that the beam line density is a linear function of time during the electron's short drifting time, $\Delta \lambda$ can be written as

$$
\Delta \lambda \approx \frac{\partial \lambda}{\partial t} \Delta t=\frac{\partial \lambda}{\partial z} c \beta \Delta t
$$

where $\Delta t$ is the drifting time. The drifting time can be estimated as the half period of the oscillation with amplitude $b$.

$$
\Delta t=\frac{1}{2} T(b)=2.0 \sqrt{\frac{\pi \varepsilon_{0} m}{\lambda e}}\left(\sqrt{2} a \arcsin \frac{1}{\sqrt{1+2 \ln (b / a)}}+\int_{a}^{b} \frac{d r}{\sqrt{\ln (b / r)}}\right)
$$

Combining Eqs. (20-22), the first part of energy gain is

$$
\Delta \mathrm{E}_{1} \approx-\beta c \sqrt{\frac{m e}{4 \pi \varepsilon_{0}}}\left(\sqrt{2} a \arcsin \frac{1}{\sqrt{1+2 \ln (b / a)}}+\int_{a}^{b} \frac{d r}{\sqrt{\ln (b / r)}}\right)\left(1+2 \ln \frac{b}{a}\right) \frac{\partial \lambda}{\partial z} \frac{1}{\sqrt{\lambda}}
$$

The secondary part of the energy gain in Eq. (19) becomes

$$
\Delta E_{2}=e \sum_{i} u_{2 i} \Delta \lambda_{i} \approx \frac{e}{2} \int u \frac{\partial \lambda}{\partial t} d t=\frac{e}{2} \frac{\partial \lambda}{\partial t} \int u d t
$$

The electron drifts from one side of the chamber wall to another side within a short time. By integrating throughout the whole drifting period, the above equation can be written as

$$
\Delta \mathrm{E}_{2}=\frac{1}{2} \beta c \sqrt{\frac{m e}{2 \pi \varepsilon_{0}}}\left(a\left[\arcsin \frac{1}{\sqrt{1+2 \ln (b / a)}}-\sqrt{2 \ln \frac{b}{a}}\right]+\frac{1}{\sqrt{2}} \int_{a}^{b} \frac{1+2 \ln (r / a)}{\sqrt{\ln (b / r)}} d r\right) \frac{\partial \lambda}{\partial z} \frac{1}{\sqrt{\lambda}}
$$


From Eqs. (23) and (25), we obtain the total energy gain when one electron hits the wall surface as

$$
\Delta \mathrm{E}=-\frac{1}{2} \sqrt{\frac{m e}{2 \pi \varepsilon_{0}}} \beta c\left(a(2 \zeta-1) \arcsin \frac{1}{\sqrt{\zeta}}+a \sqrt{2 \ln \frac{b}{a}}+\sqrt{2} \zeta \int_{a}^{b} \frac{d r}{\sqrt{\ln (b / r)}}-\frac{1}{\sqrt{2}} \int_{a}^{b} \frac{1+2 \ln (r / a)}{\sqrt{\ln (b / r)}} d r\right) \frac{\partial \lambda}{\partial z} \frac{1}{\sqrt{\lambda}}
$$

with $\zeta=1+2 \ln (b / a)$.

Figure 9 compares the energy gain given by Eq. (26) and the numerical method. The blue line is the value obtained by the numerical method and the red line is the energy gain from Eq. (26) that fits the numerical result very well. The initial electron energy when the electron is born is around a few eVs. Therefore, the electron energy when it strikes on the chamber surface is mainly decided by the energy gain from the beam. The electron energy gain at the bunch center is zero due to the zero derivation of beam profile there and it is larger around the bunch tail due to the low beam's line density around there. There are two peaks of energy gain around $550 \mathrm{~ns}$ where the derivation of the beam profile has two extrema. The maximum energy gain is $300 \mathrm{eV}$ around the bunch tail. From the electron energy at the wall surface, we can estimate the SEY. The SEY at different times also is given in Figure 9. It can be concluded from the estimated SEY that multipacting starts at $450 \mathrm{~ns}$ and it is around $550 \mathrm{~ns}$ and bunch tail due to the high energy there. Therefore, the energy gain can clearly explain when the multipacting starts and when it is strong. The mechanism of multipacting can be quantitatively described by Eq. (26).

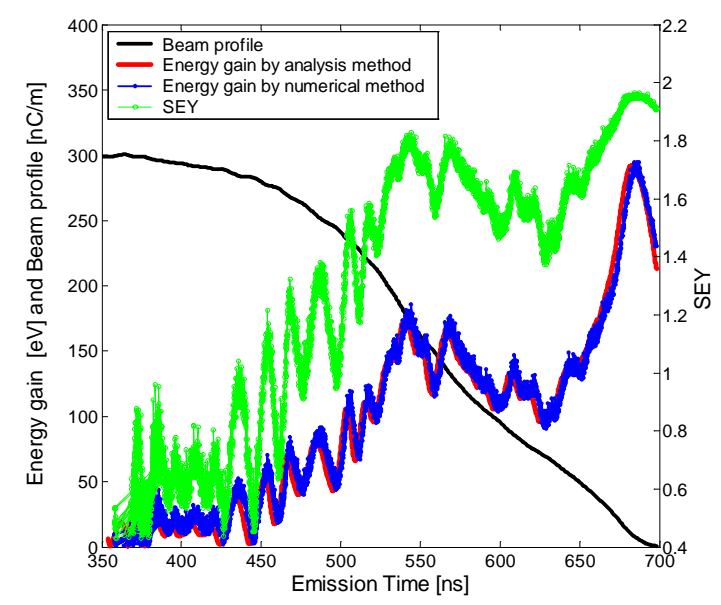

Figure 9 (Color) Comparison of energy gain. The blue line is the energy gain derived by the numerical method, the red line is the energy estimation by Eq. (26), the black line is the beam's profile, and the green line is the estimation of the SEY.

Figure 10 shows the electron density and wall current due to the electron hitting during the first 4 turns in the SNS's drift region. The electron cloud begins to build up at 500ns and strong multipacting happens at the bunch tail. This agrees with the data shown in Figure 9. During the bunch's passage, the electron line density inside the beam is almost equal to the line density inside the vacuum chamber, meaning that all electrons remain inside the beam during the bunch passage. Figure 3 and 4 explain this process. Thus, all electrons surviving from the last bunch gap will be trapped inside the beam because their oscillation 
amplitude is smaller than the beam's transverse size. These electrons interact with beam and can cause beam instabilities. On the other hand, most electrons linger around the chamber wall's surface at the bunch tail due to the strong multipacting at that moment. It is more clearly depicted in Figure 11 by the transverse distribution of the electron cloud. The electron cloud rapidly decays during the bunch gap due to the space charge effect.

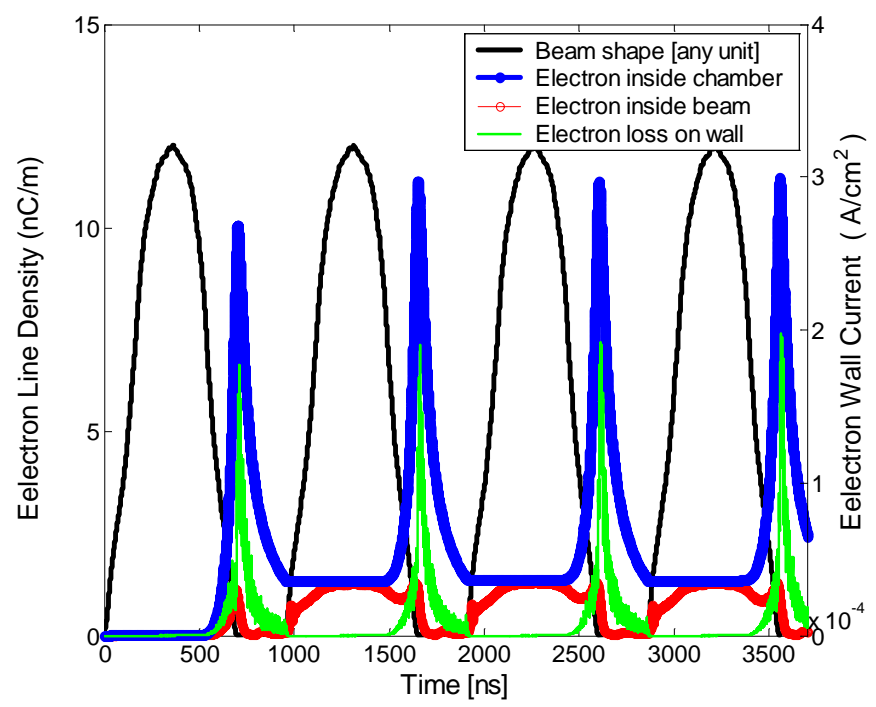

Figure 10 Electron cloud buildup in the SNS drift region. Black line shows the longitudinal beam profile. The blue line is the electron cloud line density inside chamber, which represents the total number of electrons and hence the multipacting. The red line shows the electron line density inside beam. It indicates the beam instabilities. The green line gives the wall current due to the strike of electrons. It can be used to compare with experiment result.
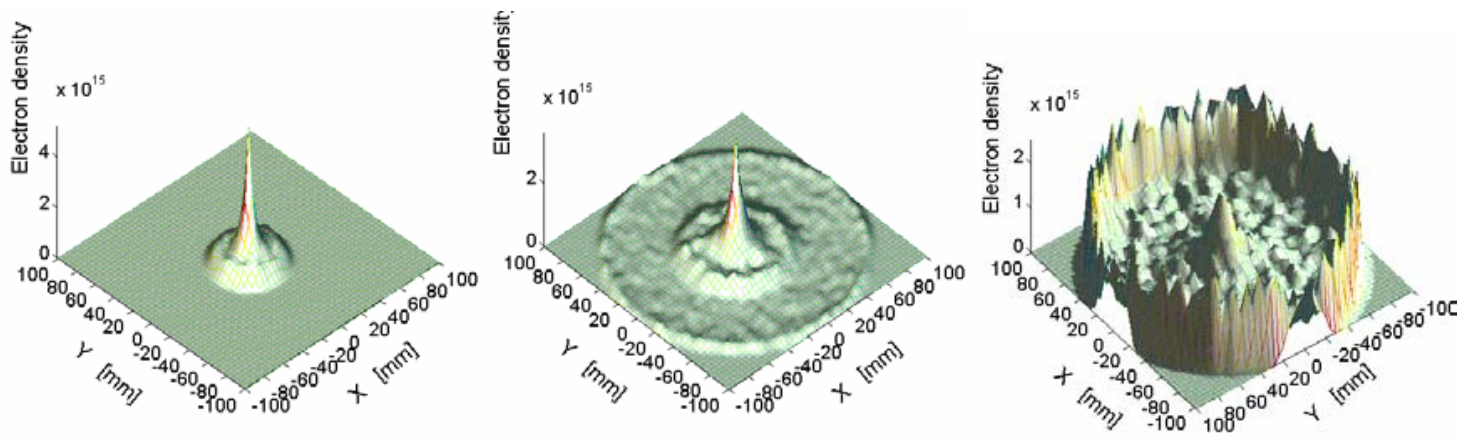

Figure 11 Electron distribution in transverse section at the bunch center (left), 210ns after bunch center (middle), and bunch tail (right).

\section{Dipole Magnetic Field}

The motion equation of a charged particle in electric and magnetic fields is

$$
m \frac{d \mathbf{v}}{d t}=e \mathbf{E}+e \mathbf{v} \times \mathbf{B}
$$


In dipole magnets, $\mathbf{B}=\left(0, B_{y}, 0\right)$, and the beam's electric field $\mathbf{E}=\left(E_{x}, E_{y}, 0\right)$. The longitudinal beam field is neglected here due to its small value. The equation of motion (27) then becomes

$$
\begin{aligned}
\frac{d v_{x}}{d t} & =\omega v_{z}+\omega E_{x} / B \\
\frac{d v_{y}}{d t} & =e E_{y} / m \\
\frac{d v_{z}}{d t} & =-\omega v_{x}
\end{aligned}
$$

where $\omega=e B / m$ is the gyration frequency in the magnet. Substituting $v_{\mathrm{x}}$ from Eq.(30) to Eq. (28),

$$
\frac{d^{2} v_{z}}{d t^{2}}+\omega^{2} v_{z}=-\omega E_{x} / B
$$

The initial conditions are given at $\mathrm{t}=0$ where $v_{x}(0)=v_{x 0}, E_{x}(0)=E_{x 0}$, and $v_{z}(0)=v_{z 0}$. With the applied initial condition, the result becomes

$$
\begin{aligned}
& v_{x}=v_{x 0} \cos \omega t+v_{z 0} \sin \omega t+\frac{\omega}{B} \sin \omega t \int_{0}^{t} E_{x} \sin \omega t d t+\frac{\omega}{B} \cos \omega t \int_{0}^{t} E_{x} \cos \omega t d t \\
& v_{z}=v_{z 0} \cos \omega t-v_{x 0} \sin \omega t+\frac{\omega}{B} \cos \omega t \int_{0}^{t} E_{x} \sin \omega t d t-\frac{\omega}{B} \sin \omega t \int_{0}^{t} E_{x} \cos \omega t d t
\end{aligned}
$$

We further integrate the expressions for $v_{\mathrm{x}}$ and $v_{\mathrm{z}}$ partially once and twice, respectively [27]

$$
\begin{aligned}
v_{x}= & v_{x 0} \cos \omega t+v_{z 0} \sin \omega t+\frac{E_{x 0}}{B} \sin \omega t+\frac{1}{\omega B} \frac{d E_{x}}{d t}-\frac{1}{\omega B}\left(\frac{d E_{x}}{d t}\right)_{0} \cos \omega t \\
& -\frac{1}{\omega B} \cos \omega t \int_{0}^{t} \frac{d^{2} E_{x}}{d t^{2}} \cos \omega t d t-\frac{1}{\omega B} \sin \omega t \int_{0}^{t} \frac{d^{2} E_{x}}{d t^{2}} \sin \omega t d t \\
v_{z}= & v_{z 0} \cos \omega t-v_{x 0} \sin \omega t+\frac{E_{x 0}}{B} \cos \omega t-\frac{E_{x}}{B} \\
& -\frac{1}{B} \cos \omega t \int_{0}^{t} \frac{d E_{x}}{d t} \cos \omega t d t+\frac{1}{B} \sin \omega t \int_{0}^{t} \frac{d E_{x}}{d t} \sin \omega t d t
\end{aligned}
$$

When the beam's space-charge field changes slowly compared with the gyration frequency, i.e., when

$$
\left|\frac{1}{\omega E_{x}} \frac{d E_{x}}{d t}\right|<<1,
$$

the integrals in Eqs. (34-35) become very small and can be neglected. In the normal dipole magnet of the SNS ring, the gyration frequency is about $20 \mathrm{GHz}$ with small gyration radius less than $0.01 \mathrm{~mm}$ where Eq. (36) is satisfied. Using the substitution 


$$
\begin{aligned}
& v_{z}^{\prime}=v_{z}+\frac{E_{x 0}}{B}, \\
& v_{z 0}^{\prime}=v_{z 0}+\frac{E_{x 0}}{B},
\end{aligned}
$$

we find that the motion in the present approximation will consist of a gyration with velocity $v^{\prime}$ superimposed on a translation with drift velocity $\mathbf{E} \times \mathbf{B} / B^{2}$ that varies slowly with time. The kinetic energy of gyration motion depends on the initial condition when the electron is born, which is around a few eVs. The cross-field drifting energy at the wall's surface of the dipole magnet is less than $10 \mathrm{eV}$ in the SNS, where the peak beam electric field is about $10 \mathrm{kV} / \mathrm{m}$ and magnetic field is $0.8 \mathrm{~T}$. As shown in Eq. (29), vertical motion is independent. An electron may receive more energy from the beam due to the beam's vertical space-charge field. Following the same procedure, as an electron moves radially in the drift region, we can assess the energy gain in a dipole magnet for an electron moving along the vertical magnetic-field line located at horizontal coordinate $\mathrm{X}$ as

$$
\begin{aligned}
& \Delta E=-c \beta \sqrt{\frac{m e}{2 \pi \varepsilon_{0}}} \frac{\partial \lambda}{\partial z} \frac{1}{\sqrt{\lambda}}\left(1-\frac{\mathrm{X}^{2}}{\mathrm{a}^{2}}+\ln \frac{\mathrm{b}^{2}}{\mathrm{a}^{2}}\right)\left(a G+\int_{\sqrt{a^{2}-X^{2}}}^{\sqrt{b^{2}-X^{2}}}\left(\ln \frac{b^{2}}{X^{2}+y^{2}}\right)^{-1 / 2} d y\right) \\
& +\frac{1}{2} c \beta \sqrt{\frac{m e}{2 \pi \varepsilon_{0}}} \frac{\partial \lambda}{\partial z} \frac{1}{\sqrt{\lambda}} \int_{0}^{\sqrt{a^{2}-X^{2}}} \frac{y^{2}}{a^{2}}\left[\ln \left(1-\frac{\mathrm{X}^{2}}{\mathrm{a}^{2}}+\ln \frac{\mathrm{b}^{2}}{\mathrm{a}^{2}}+\frac{y^{2}}{a^{2}}\right)\right]^{-1 / 2} d y \\
& +\frac{1}{2} c \beta \sqrt{\frac{m e}{2 \pi \varepsilon_{0}}} \frac{\partial \lambda}{\partial z} \frac{1}{\sqrt{\lambda}} \int_{\sqrt{a^{2}-X^{2}}}^{\sqrt{b^{2}-X^{2}}}\left(1-\frac{X^{2}}{\mathrm{a}^{2}}+\ln \frac{X^{2}+y^{2}}{a^{2}}\left[\ln \left(\frac{b^{2}}{X^{2}+y^{2}}\right)\right]^{-1 / 2} d y\right. \\
& \left.\Delta E=-c \beta \sqrt{\frac{m e}{2 \pi \varepsilon_{0}}} \frac{\partial \lambda}{\partial z} \frac{1}{\sqrt{\lambda}}\left[\frac{b^{2}}{X^{2}} \int_{0}^{\sqrt{b^{2}-X^{2}}}\left(\ln \frac{b^{2}}{X^{2}+y^{2}}\right)^{-1 / 2} d y-\frac{1}{2} \int_{0}^{\sqrt{b^{2}-X^{2}}} \frac{X^{2}+y^{2}}{X^{2}}\left(\ln \frac{b^{2}}{X^{2}+y^{2}}\right)^{-1 / 2} d y\right] \quad|\mathrm{X}|>\mathrm{a}\right) \\
& \text { where } G=\arcsin \left[\frac{\sqrt{a^{2}-X^{2}}}{a}\left(1-\frac{X^{2}}{a^{2}}+\ln \frac{b^{2}}{X^{2}+a^{2}}\right)^{-1 / 2}\right] \text {. }
\end{aligned}
$$



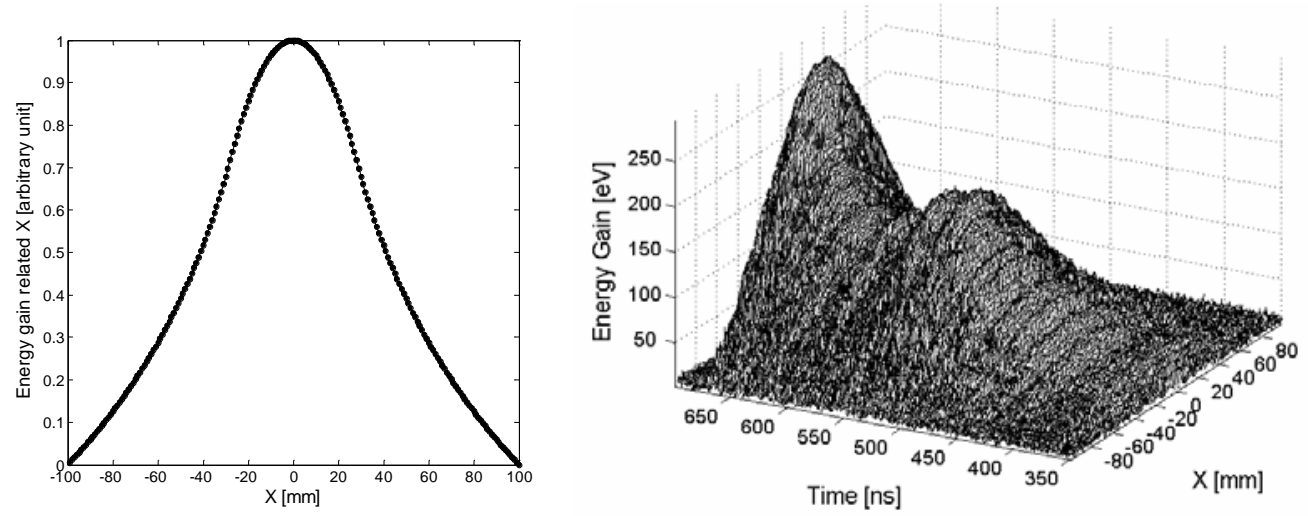

Figure 12 Energy gain at the wall surface for different X-coordinates. The left plot shows the electron energy gain as a function of the horizontal coordinate. It is normalized by the peak energy gain at the chamber's center $X=0$. The right plot shows the energy gain of direct drifting electrons in the SNS's dipole magnets with $\mathrm{B}_{\mathrm{y}}=7935$ Gauss.

\section{IMPORTANT FACTORS IN MULTIPACTING AND BUILD-UP}

Multipacting strongly depends on the energy of the electron when it hits the vacuum wall's surface. Accordingly, multipacting is related to the particle's motion. Based on the motion of the electron, a few important factors on multipacting and electron cloud build-up that we studied are discussed in the following paragraphs.

\section{Effects of the Longitudinal Beam Profile and Bunch Length}

The effect of the longitudinal beam profile on the electron's energy gain is shown in Eq. (26). The energy gain is governed by the longitudinal beam's profile factor

$$
\text { Factor }_{\text {profile }}=-\frac{\partial \lambda}{\partial z} \frac{1}{\sqrt{\lambda}}
$$

The first part of the profile factor, the derivation of the line density, represents the difference of beam density between the moments of electron emission and of electron loss. The smaller the beam profile derivation, the smaller is the electron's energy gain. Drifting time represents the secondary part in the profile factor. It usually entails a longer drifting time round the bunch tail due to the low density of the beam there, and hence, a bigger energy gain. This is the mechanism whereby strong multipacting always happens at the bunch's tail, as shown in Figure 9. The effect of the longitudinal beam profile is at first included analytically in the form of derivative of electron oscillation, without further discussion [16]. This effect has been studied in experiments at the PSR [28] and by simulation [17]. The derivative of the longitudinal beam profile was noticed to be important at that time. However, the effect of drifting time, which is the most notable mechanism of multipacting, remains unknown.

Adjusting the buncher's phase can change the bunch tail. In the PSR, the electron signal at the bunch tail increases $140 \%$ when the rf of the buncher phase changes from $281^{\circ}$ to $301^{\circ}$ due to the increasing tail 
shoulder [28]. However, the instability threshold simultaneously increases by only 26\% [29]. Figure 13 shows the simulated electron-cloud density inside the chamber for two assumed nonsymmetrical longitudinal beam profiles. The longer tail causes stronger multipacting at the bunch's tail. On the other hand, the electron clouds inside the beam during the passage of the bunch of these two profiles, proportional to the rate of growth of proton instability [30], closely resemble each other because of the faster decay in the stronger multipacting case. Therefore, the measured electron signal at bunch tail is sensitive to the bunch phase, but the instability is not.

Using the same beam profile as in Figure 9, Pivi and Furman [17] artificially truncated the bunch tail while maintaining the same integrated beam charge. The result of their simulation shows that the electron density can be reduced by a factor more than 100 when the beam profile is cut at 500ns. Comparing this finding with Figure 9, cutting the bunch at 500 ns will cut off most of the multipacting area. Figure 9 explains these phenomena.

Figure 14 compares three types of assumed beam profile; Gaussian, sinusoidal, and elliptical . They all have the same integrated beam charge and secondary emission parameters as shown in Table 1 and 2. The figure also has the energy gain and the SEY. Comparing the energy gain and SEY of these three distributions, the Gaussian profile is the worst. Multipacting happens at 375ns, just $25 \mathrm{~ns}$ after the bunch center and the SEY is almost a constant value close to 2 for long time. The elliptical profile is the best, with multipacting starting later at $600 \mathrm{~ns}$ and a smaller SEY. Therefore, the Gaussian profile has the largest peak electron density, $150 \mathrm{nC} / \mathrm{m}$, while the elliptical profile has the minimum, of $1 \mathrm{n} \mathrm{C/m}$, as shown in Figure 14 (d). A realistic beam profile gives an electron density of about $12 \mathrm{nC} / \mathrm{m}$. It is a little worse than the sinuous profile, which gives an electron density $8 \mathrm{nC} / \mathrm{m}$. All these findings can be explained by the beam profile factor in Eq. (40). Therefore, the beam longitudinal profile pays a very important role in the multipacting of the long bunched beam.

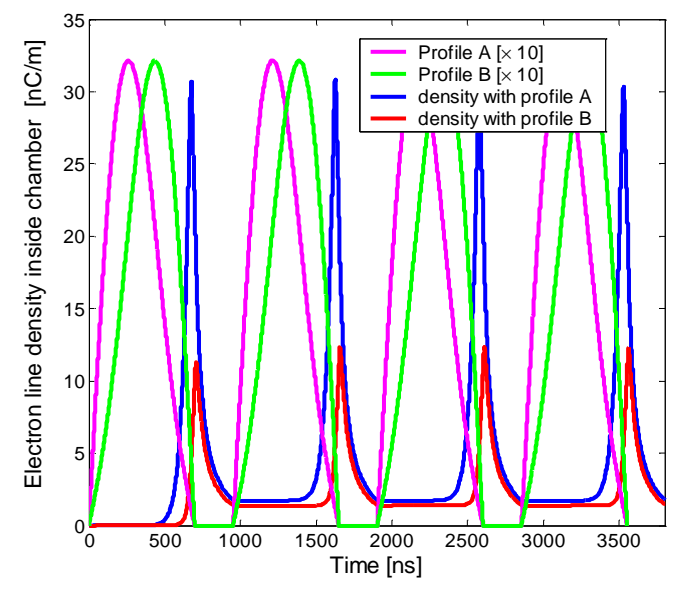

Figure 13 Electron line densities inside the chamber for beam profiles with different bunch tails. 


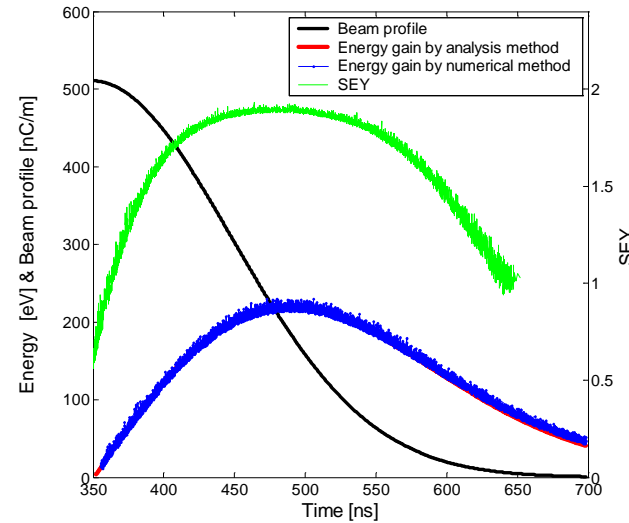

(a)

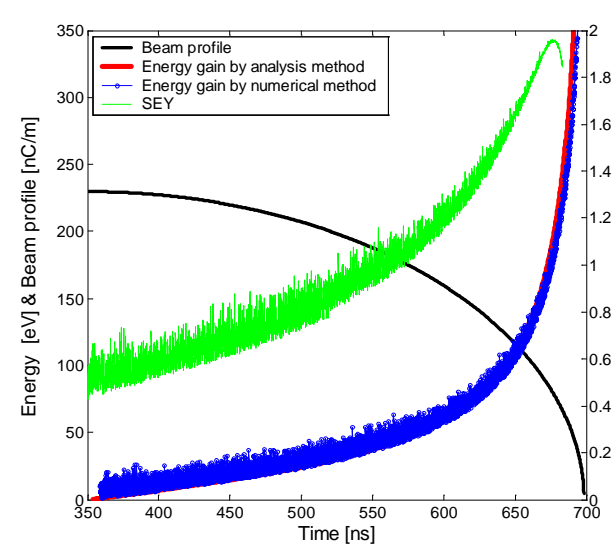

(c)

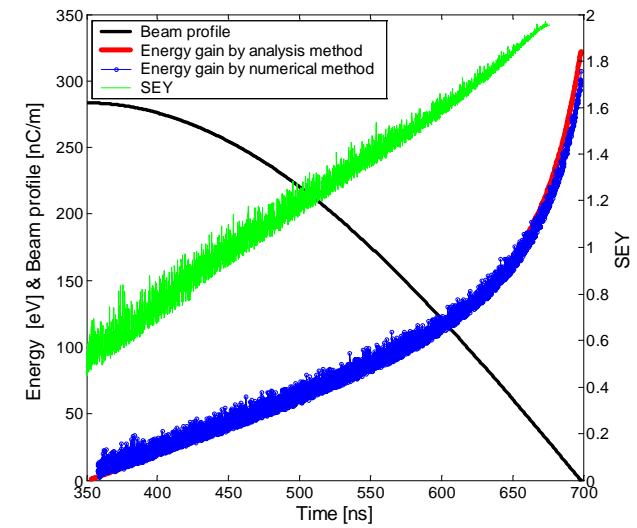

(b)

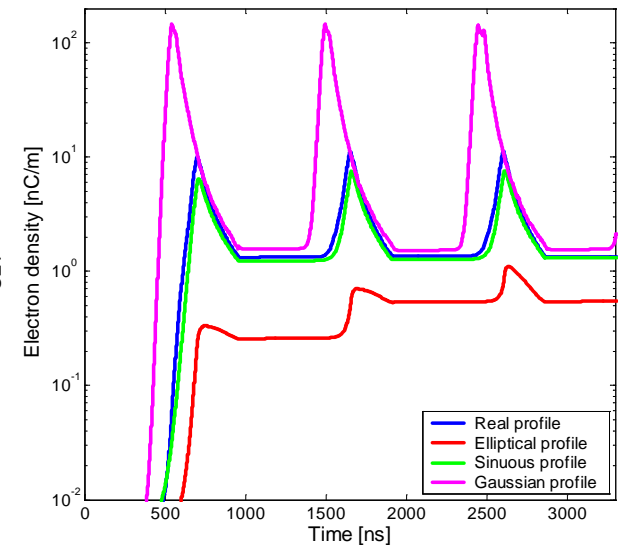

(d)

Figure 14 Comparison the effects of a Gaussian, sinuousoidal, elliptical, and realistic beam profile.

Using the same secondary-electron parameters, Figure15 gives the PSR beam profile, simulated electron energy gain, SEY, and electron cloud build-up in the PSR's drift region. We note that the distribution in the transverse plane of the PSR beam is Gaussian. The electrons inside the beam must fall within $\sqrt{3}$ root mean square (RMS) of the beam's size in order to be consistent with its cylindrical shape. In plotting Figure 15 , the longitudinal beam profile factor was multiplied by a constant factor to compare its shape with the energy gain. The energy gain still agrees well with the longitudinal beam profile factor's shape because Gaussian and uniform transverse-beam profiles do not make any difference in the electron's energy gain for the same RMS size, as discussed later. The figure clearly shows that strong multipacting could occur early, just $20 \mathrm{~ns}$ after the bunch's center. The PSR beam is shorter than the SNS beam, and its total multipacting time is about one times less. However, it has bigger SEY due to the effects of the beam profile. As a result, both beams have almost the same electron cloud density. Note that the same SEY parameters shown in table 2 are used for both the SNS and PSR beam.

Figure 16 shows the measured electron's signal at LANL PSR [31]. The number of electrons grows dramatically at the trailing edge of the proton bunch and it is peak at the bunch tail. This is consistent with the shape of simulated electron wall current (Green line in Figure 15b) and can be clearly explained by the 
electron energy gain (Blue line in Figure 15a). The measured electron energy at the wall is up to $300 \mathrm{eV}$, which roughly agrees with the simulated number $200 \mathrm{eV}$. The discrepancy in electron energy at the wall may come from the difference of the parameters between the simulation and experiment. The wall current due to electron hitting is $0.4 \mathrm{~mA} / \mathrm{cm}^{2}$ determined experimentally [4], and is $0.6 \mathrm{~mA} / \mathrm{cm}^{2}$ by simulation, as shown in Figure 15(b).

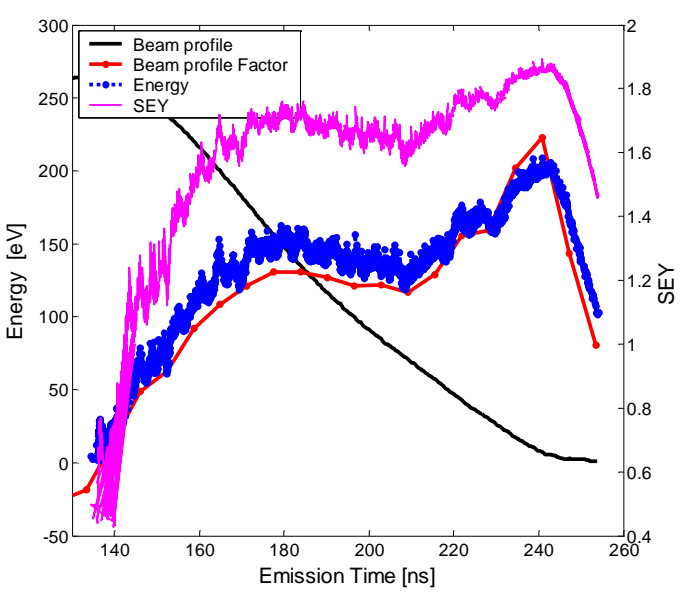

(a)

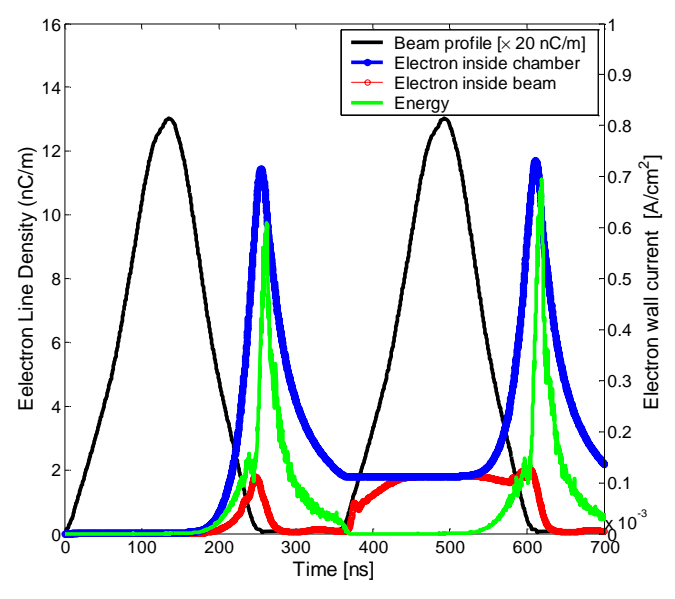

(b)

Figure 15 Energy gain (a) and electron cloud build-up (b) in the PSR drift region

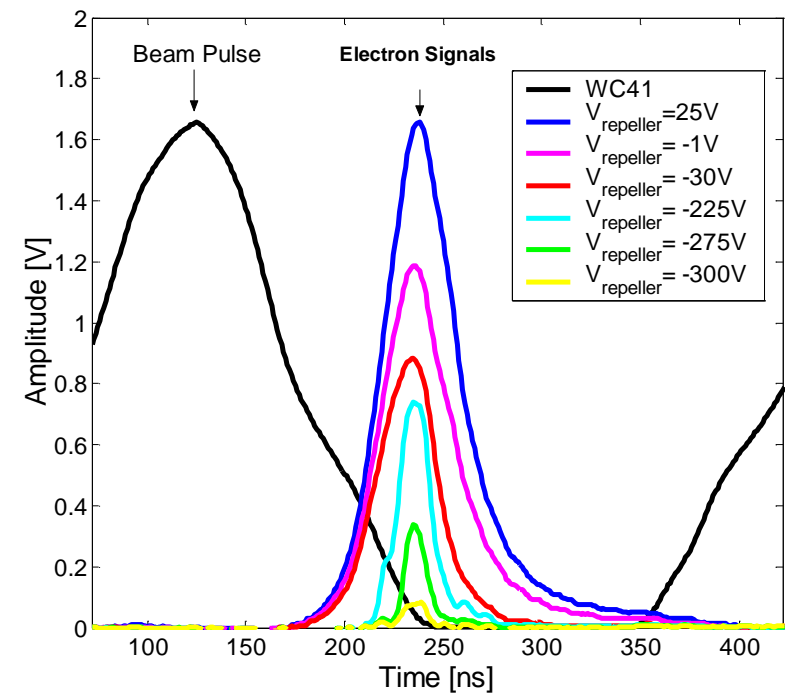

Figure 16 Electron signals measured at the PSR as a function of time relative to the proton-beam pulse during a single revolution. The repeller voltage $V_{\text {repller }}$ is varied to select the electrons striking the detector according to their energy, $8 \mu \mathrm{C} /$ pulse beam.

For a given longitudinal beam profile, the electron density inside chamber slowly changes with the bunch's length provided that the particle density inside the bunch is kept constant by maintaining the bunch's intensity proportional to its length. A long bunch reduces the electron's energy gain but it may increase the possible multipacting time.

If bunch length is reduced, and its intensity kept constant, the electron density inside chamber during the gap will increase quickly with a decrease in bunch length due to both a high gain in energy and fast 
multipacting frequency for short bunch. However, fewer electrons survive from the last bunch gap when the bunch is shorter due to the stronger space-charge force of the electron cloud at the gap, and the long bunch gap for a short bunch. Therefore, a short bunch causes a strong multipacting at its tail and low electron density inside the beam. Consequently, a short bunch is a more stable one. This agrees with the results of a PSR beam study finding that a higher beam current can be stored with shorter bunch length at the same instability threshold.

\section{The Beam's Transverse Profile and Beam Size}

Figure 17 plots the build up of the electron cloud and the energy gain for cylindrical beam and Gaussian beam with the same sized RMS in the SNS's drift region. The two transverse profiles exhibit very similar electron densities inside the beam and inside the chamber. The difference in density inside the chamber is less than 5\%, while it is equal inside the beam. The underlying explanation is that the space-charge force does not depend on the transverse spatial charge distribution of the beam for a given RMS size [32]. This is confirmed by the electron-energy gain, which is the same for cylindrical beam and Gaussian beam (Figure 17). Therefore, the electron-energy gain of a Gaussian beam can be estimated with the formula used for a cylindrical uniform beam with the same RMS size, as given by Eqs. (26) and (39).
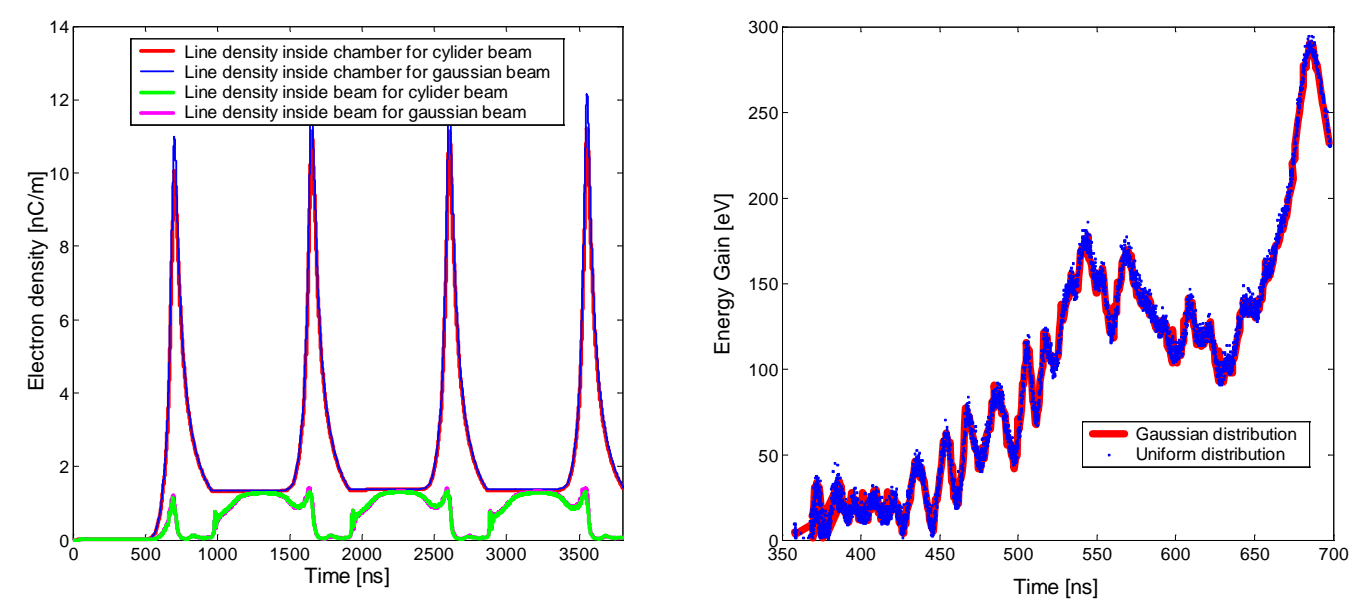

Figure 17 Effect of the transverse beam profile on electron density (left) and electron energy gain (right) in the SNS drift region

Although the gain in electron energy gain is independent of the beam's transverse profile, the azimuthal distribution of the electron cloud is related to the transverse profile's shape: there is more of the electron cloud in the orientation of the larger beam's size. Figure 18 is a simulated electron-cloud distribution in the transverse plane for an assumed SNS's flat beam $\sigma_{x}: \sigma_{y}=2: 1$ at different times. The space-charge force in the direction of the larger beam size is stronger and it confines into electrons moving along this direction, and then stronger multipacting occurs there. Browman observed a similar phenomenon in the LANL PSR [33]. There is stronger electron signal in the larger betatron function direction. As a result, the electron cloud may different in horizontal and vertical direction and hence the beam instabilities. 

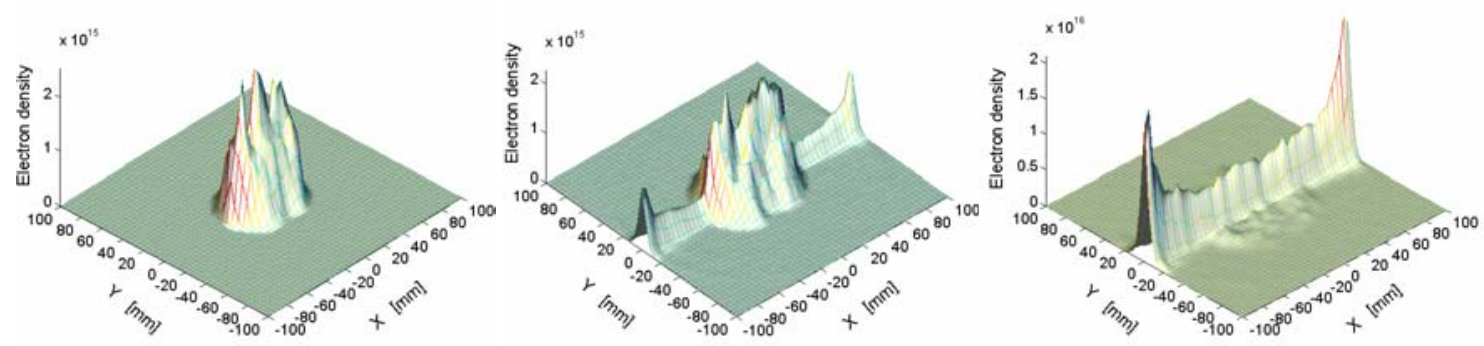

Figure 18 Transverse distribution of electron cloud for an assumed SNS flat transverse beam profile with $\sigma \mathrm{x}: \sigma \mathrm{y}=2: 1$ at 350ns (left), 560ns (middle), and $630 \mathrm{~ns}$ (right).

The beam size's effect on the energy gain in Eq.(26) is shown in Figure 19(a). A smaller beam size contributes to stronger space-charge field as shown in Eq. (5), and hence, larger electron-energy gain and stronger multipacting. Figure 19(b) demonstrates the effect of the beam's transverse size on the peak electron-cloud line density inside the beam chamber and the average volume density inside the beam. The density inside the chamber is roughly inversely proportion to the transverse beam size. It scales as

$$
\lambda_{\text {chamber }}[n C / m]=21-0.27 \mathrm{a}[\mathrm{mm}]
$$

However, the electron volume density inside beam is scaled as

$$
\rho_{c e n}\left[\mathrm{nC} / \mathrm{cm}^{3}\right]=4.9 e^{-0.1 a[\mathrm{~mm}]} .
$$

The volume density inside the beam exponentially decreases with the transverse size of the beam. Therefore, a big beam size is very helpful in reducing beam instabilities caused by the electron cloud. This is consistent with the PSR experimental study [29] wherein the instability threshold rose by a factor two when the beam size was increased from $15 \mathrm{~mm}$ to $34 \mathrm{~mm}$.

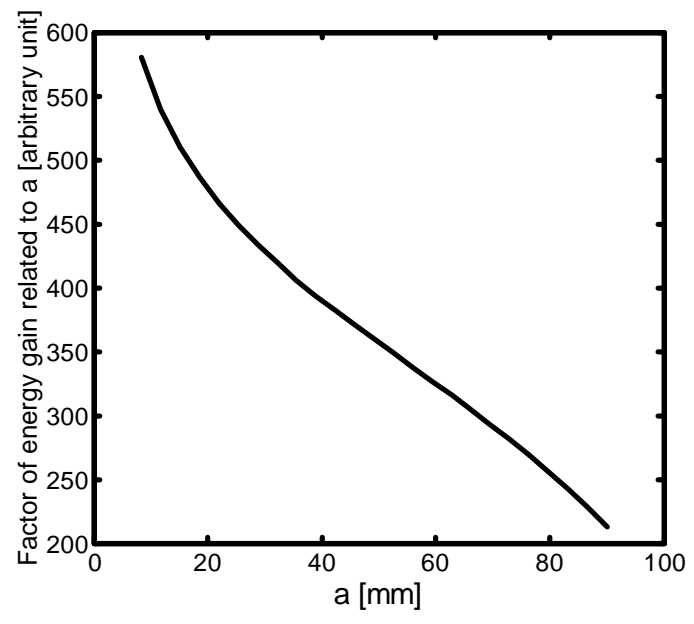

(a)

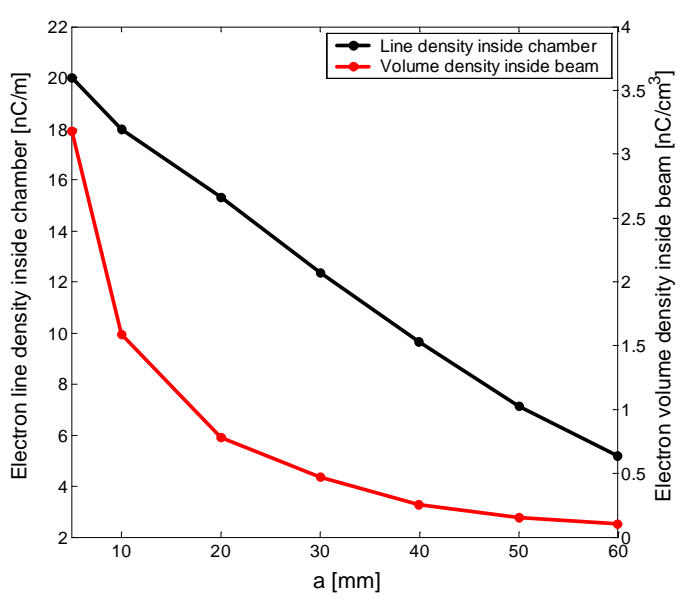

(b)

Figure 19 Effect of beam size on electron energy gain(a), and electron cloud density in the SNS drift region(b) 


\section{Effects of the Beam's Intensity}

For a fixed longitudinal beam-profile shape, the energy gain calculated with Eq. (26) is proportional to the square root of the beam's intensity $N$. Figure 20(a) shows the energy gain for the SNS beam, evaluated by the numerical method, for various intensities. The first peaks in the figure are $119 \mathrm{eV}, 168 \mathrm{eV}$, and $205 \mathrm{eV}$ that correspond to $N=1.0 \times 10^{14}, N=2.0 \times 10^{14}$, and $N=3.0 \times 10^{14}$. They agree well with Eq. (26) .

We kept the number of protons lost at the same value for different beam intensities to check the latter alone; Figure 20(b) shows the corresponding build-up of electron line density inside the chamber. At high beam density, the density of the electron cloud increases quickly with increments of the beam's intensity, thereby predicating that the former is very sensitive to the latter. This phenomenon can be explained by two mechanisms. One is the plot of energy gain shown in Figure 20(a) showing that a strong beam causes a larger energy gain and hence, a larger SEY. Another more important mechanism is the higher multipacting frequency for a stronger beam. The drifting time is inversely proportional to $\sqrt{\lambda}$. Therefore, a more intense beam contributes to a higher multipacting frequency. In the case of $N=2 \times 10^{14}$, the maximum number of electrons inside the chamber is $2.2 \times 10^{13}$ and the total number of primary electrons is $2 \times 10^{8}$. Therefore, on average, one primary electron can produce $1 \times 10^{5}$ secondary electrons at the end of a bunch. Assuming a constant SEY of 2.0, then one primary electron hits the chamber wall about $\log _{2}\left(1 \times 10^{5}\right)=16$ times with a yield 2.0 during the passage of the whole bunch. If the beam's intensity increases to $N^{\prime}$, the times of multipacting per electron will become $16 \sqrt{N^{\prime} / N_{0}}$ with $N_{0}=2.0 \times 10^{14}$. In this way, we can estimate the electron-cloud density for different beam intensities due to the multipacting-frequency effect. Figure 20(c) shows the relationship of the electron cloud's line density inside the chamber and the beam's intensity obtained by simulation and the estimation method above. They are very close when the beam's intensity is small. However, the discrepancy becomes bigger when the beam intensity increases due to the space-charge effect. We point out that we did not include in the estimation the effect of beam intensity on electron-energy gain and the space-charge effect. By fitting the simulation result, we get the scaling law of electron density with beam intensity as

$$
\lambda_{\text {chamber }}[n C / m]=78-112 \times 1.0^{-14} N+39 \times 1.0^{-28} N^{2}
$$

The combined effects of multipacting frequency, energy gain, and the space-charge force causes electron density to grow with the increase in the beam's intensity. In the PSR, the measured electron-cloud signal shows a similar strong dependence on beam intensity [4].

On the other hand, the density of the electron cloud inside the beam becomes saturated, or even may decrease at high beam intensities, as shown in Figure 20(d). When bunch intensity increases, the electron number inside the chamber during the bunch gap also rises, and hence, the space-charge field. The stronger space-charge force entails a short decay time for the electron cloud during the bunch gap. As a result, a high beam intensity affects the electron number inside the beam chamber in two ways: increasing it by stronger multipacting, and reducing it by promoting a quicker decay at the bunch gap due to the stronger space charge. We note that the number of electrons inside the beam roughly equals the number of electrons that 
survive from the last bunch gap due to the beam field's trapping effect (Figure 4 and 10). This mechanism can explain the saturation or decrease of electron density inside the beam when very strong multipacting takes place.

In LANL PSR, we measured the electron signal when the electrons strike the chamber wall surface, which corresponds to the simulated wall current in Figure 20(d). It is called prompt electron signal. An electron sweeping detector was developed as a diagnostic to measure electrons lingering inside the pipe[34]. Basically it is an RFA with an electrode opposite the RFA. The electrode is pulsed with a short fast pulse (up to $1 \mathrm{kV}$ ) to sweep low energy electrons at the bunch gap from the pipe into the detector. We call them swept electrons, which corresponds to the simulated surviving the electrons from the bunch gap. Figure 21 shows the measured prompt electron signal and swept electron signal with different bunch intensity in PSR [35]. The prompt electron signal increase without saturation. However, the swept electron signal saturated at high beam intensity. The increase in the growth of electron cloud density inside the beam and the rise in the electron wall current with an increase in beam intensity, as shown in Figure 20(d), qualitatively agree with the experimental results at the PSR (Figure 21). Both the experiment and simulation conclude that the beam instabilities saturate at high beam intensity, but the multipacting does not.

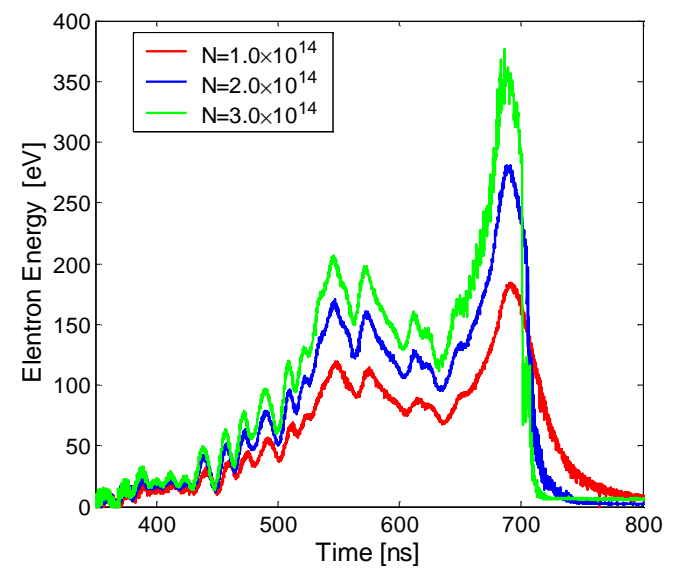

(a)

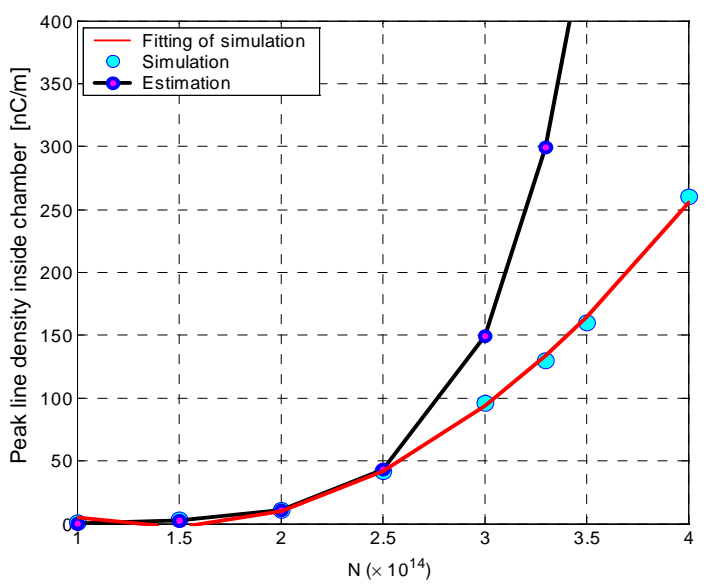

(c)

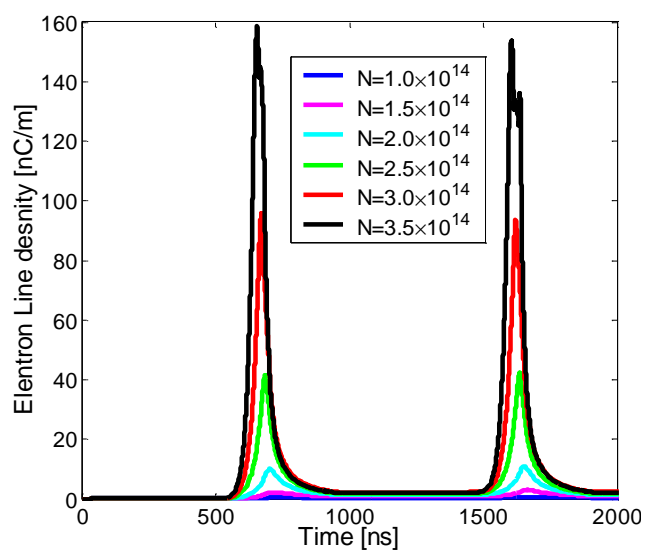

(b)

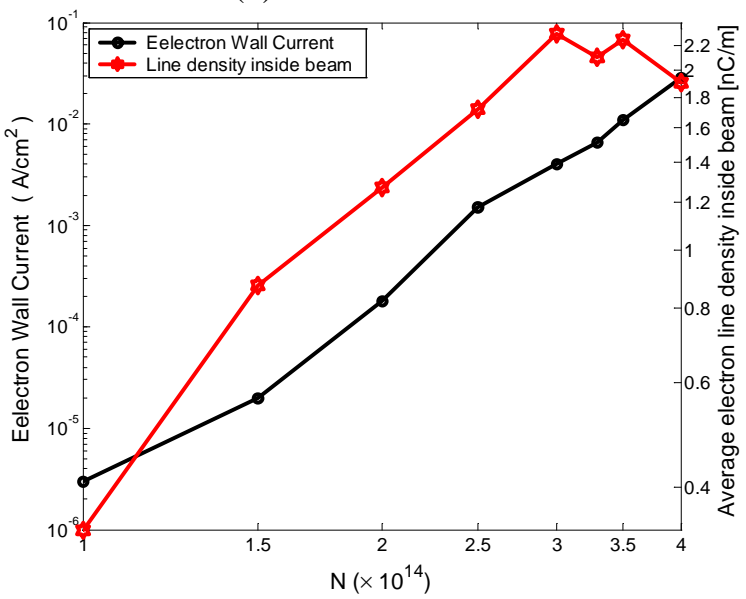

(d) 
Figure 20 Beam intensity effects on energy gain (a), electron cloud buildup (b), peak electron density inside the chamber (c), electron wall current, and, average line density inside beam (d) in the SNS drift region

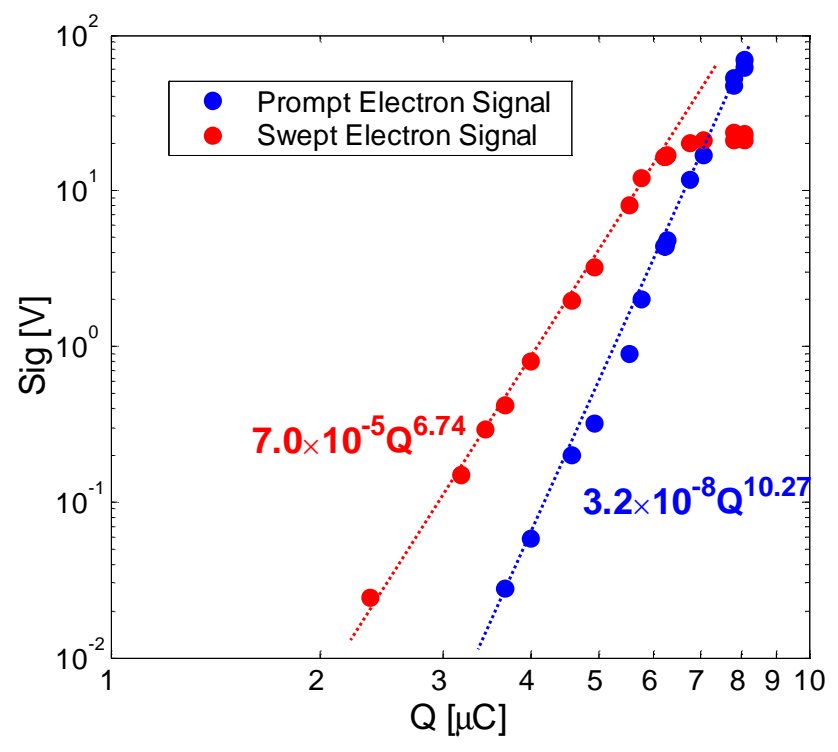

Figure 21 Measured prompt electron and swept electron signal amplitudes are plotted as a function of stored beam intensity in LANL PSR. All other beam parameters were fixed including buncher voltage and accumulation time.

\section{Bunch Gap}

The deep trapping effects of the surviving electrons from the last bunch gap play a major role in the operation of the long bunch machine. According to Eq. (14) and Figure 3 and 4, electrons surviving after the bunch gap can be soundly trapped inside the beam until the end of the passage of the next bunch. Electrons inside the beam are the main source of electron-proton instabilities [30]; electrons outside the beam have little effect upon the beam's dynamics. Although strong multipacting occurs at the bunch tail, most electrons remain outside the beam, as shown in Figure 11. The electrons' density inside the beam at the bunch tail is at the same level as at the other times shown in Figures 10 and 15. Therefore, surviving electrons from the last bunch gap cause bunch instabilities.

Note that the bunch gap has a very weak effect on the peak electron line density inside the chamber due to the single-bunch multipacting mechanism. Therefore, the peak electron line density inside the vacuum chamber is almost the same during the passage of the first turn and of the following turns (Figures 10 and 15). However, the bunch gap contributes to reducing the electron density inside the beam. If the gap is long enough, compared with the decay time of the electron cloud during the gap, the electron density inside the beam will be lowered significantly, and hence, the beam's instabilities also. When the bunch gap is short such that the electron cloud cannot decay to zero by the end of the gap, a clearing electrode can be applied to remove the electron cloud. A weak clearing field is very helpful in reducing the number of electrons inside the beam during the bunch's passage.

The protons remaining at the bunch gap due to their wider momentum spread and a large pulse width can slow down electrons loss at the gap because of its space-charge effect. The percentage of protons at the gap 
is less than $1 \times^{-4}$ for the SNS design beam. The simulated electron line density inside the chamber increases $18 \%$ and $33 \%$, respectively, for $1 x^{-4}$ and $1 x^{-2}$ protons at the gap. However, the electron density inside the beam increases $30 \%$ and $300 \%$, respectively, due to their slow decay during the gap. Because the growth rate of the beam's instability is proportional to the electron density inside the beam, it follows that instability is highly sensitive to the beam at the gap, even though that parameter itself has a weak effect on the average electron density inside the chamber.

\section{Effects of Chamber Size}

Figure 22 (a) shows that the energy gain in Eq. (26) is almost a linear function of the vacuum chamber's size $b$. The physics underlying this relationship comes from the drifting time derived in Eq. (22). A large chamber imposes a long drifting time, and hence, larger gains in energy. Figures 22(b) and (c) show the simulated energy gain and electron cloud buildup for chambers of different sizes. The energy gain is a linear function of $b$, which agrees with Eq. (26). The SEY is very different for the $b=5 \mathrm{~cm}$ and $10 \mathrm{~cm}$ cases; however, the difference becomes smaller when $b$ increases further because the SEY varies slowly when the incident energy is closer to the energy at peak SEY (Figure 1). It is interesting that the electron density inside the chamber rises with the increment of $b$ and then decreases if $b$ increases further. Two rules govern the character of the electron density with $b$. A larger $b$ contributes to higher energy and hence, a larger SEY. However, the electrons' drifting time also is longer for a larger $b$. Consequently, a larger $b$ gives a larger SEY and lower multipacting frequency. Note that while SEY is not sensitive to $b$ when $b$ is large enough, the multipacting frequency is. Therefore, the electron density has maximum value for the median $b$. For the SNS, this value is $10 \mathrm{~cm}$, which is exactly the radius of the SNS's design chamber. However, the electron density inside the beam increases with the size of $b$ and saturates at around $15 \mathrm{~cm}$, as seen in Figure 22(c).

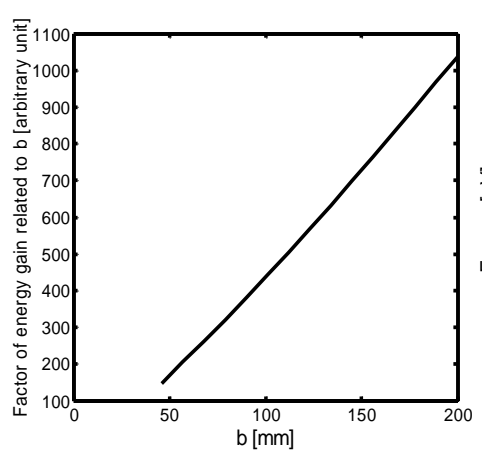

(a)

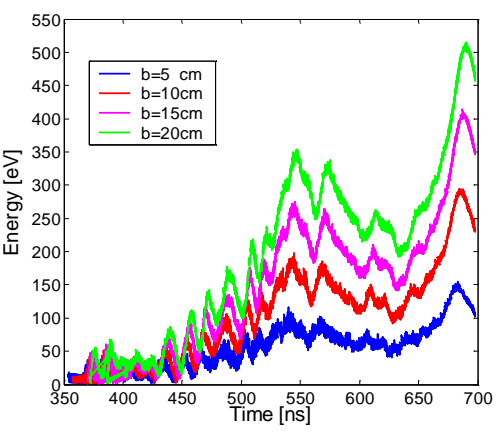

(b)

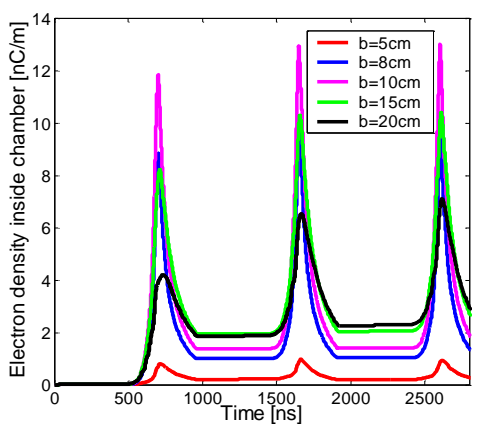

(c)

Figure 22 Effect of chamber size on energy gain (a), energy gain for different chamber sizes (b), and, the buildup of the electron cloud density inside the chamber (c) in the SNS drift region with a constant beam size

In a real machine, the beam chamber is big when the size of its local beam is large. In general, the ratio of beam chamber's size to that of the beam is roughly constant along the storage ring. Therefore, both beam 
chamber and beam size vary along the ring. Figure 23 plots the electron density for different sizes of chambers while keeping the ratio of $b$ to $a$ constant. The electron-cloud line density inside the chamber has peak at the median of $b$. However, the volume density inside the beam decreases linearly with $b$. Therefore, employing a large-sized beam and chamber can reduce the beam's instabilities.

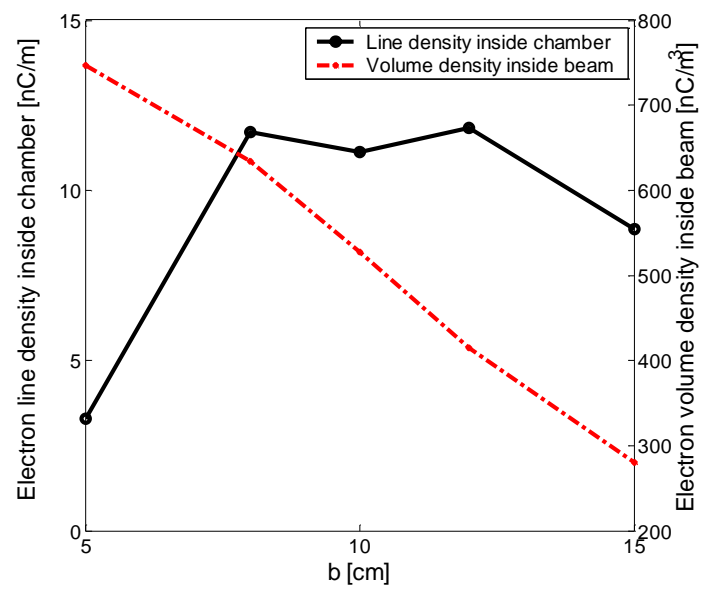

Figure 23 Electron cloud density for different sizes of chambers with a constant $b / a$

\section{Peak SEY and Energy at Peak SEY}

It is well known that the density of the electron cloud is very sensitive to peak SEY when multipacting occurs due to its exponential growth. In the absence of a space-charge effect, electron density should increase exponentially with SEY. Figure 24 shows the electron build-up and electron density for different peak SEYs. Electron density inside the chamber increases linearly with peak SEY, at a rate that is slower than the exponential growth due to the space-charge effect. In contrast, the average volume electron density inside the beam approaches saturation for a big peak SEY due to the strong space-charge effect. Because beam instability is governed primarily by volume density inside beam, we conclude that the beam's instabilities will saturate at certain peak SEY. However, the heat-load caused by the electron-cloud hitting the chamber does not saturate until the peak SEY is 2.5.

The electron energy gain with a long beam, which usually is less than the energy at peak SEY, is much smaller than that with short bunch, such as in B-Factories. Accordingly, a long beam is more sensitive to the energy at peak SEY. The energy at peak SEY has equivalent effects as the peak SEY. Figure 25 shows the electron build-up and electron density for different energies at peak SEY. Both the electron line density inside the chamber and the electron volume density inside the beam increase linearly with the decrement of energy at peak SEY. The electron volume density inside the beam does not reach saturation because the electron line density inside the chamber is not large enough. For the SNS beam, if the energy at peak SEY in Table 2 falls from $330 \mathrm{eV}$ to $246 \mathrm{eV}$, the electron density inside chamber will increase from $12 \mathrm{nC} / \mathrm{m}$ to $67 \mathrm{nC} / \mathrm{m}$. The effect is the same as increasing the SEY from 1.74 to 2.07. However, the effect on electron 
density inside beam is stronger than increasing the SEY from 1.74 to 2.5. Therefore, a bigger energy at peak SEY can significantly reduce the beam's instability.
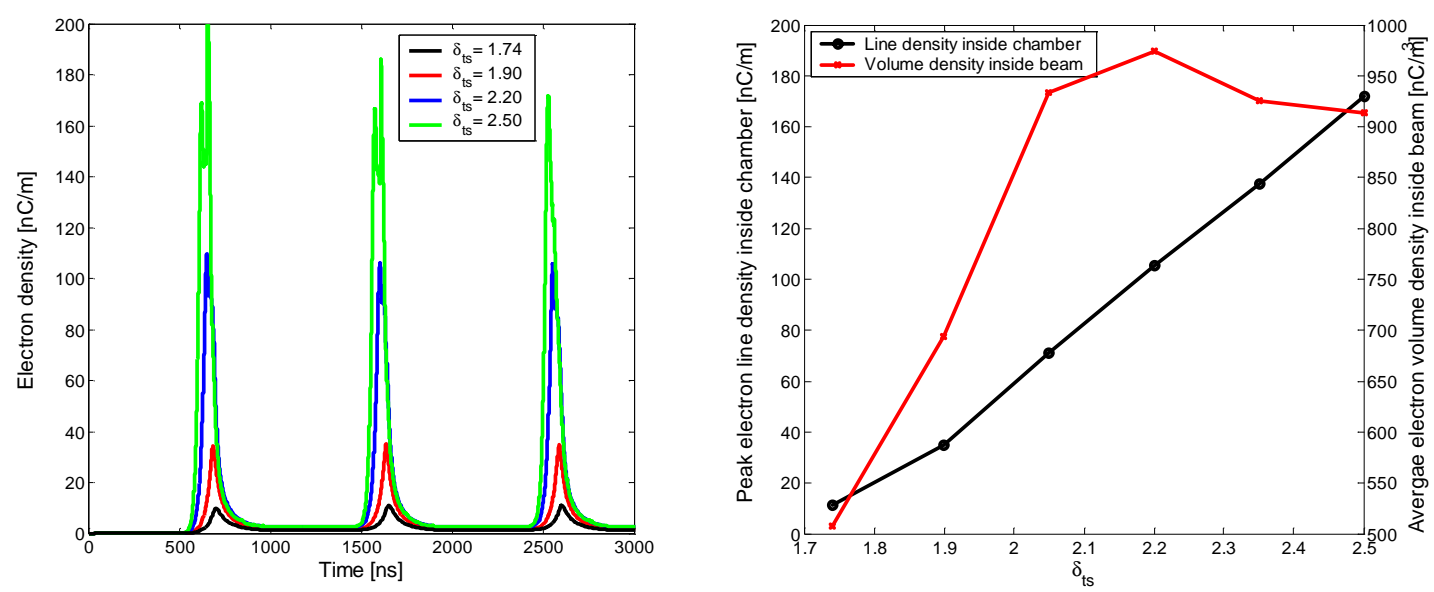

Figure 24 The effects of peak SEY on electron build-up (left) and electron density (right).
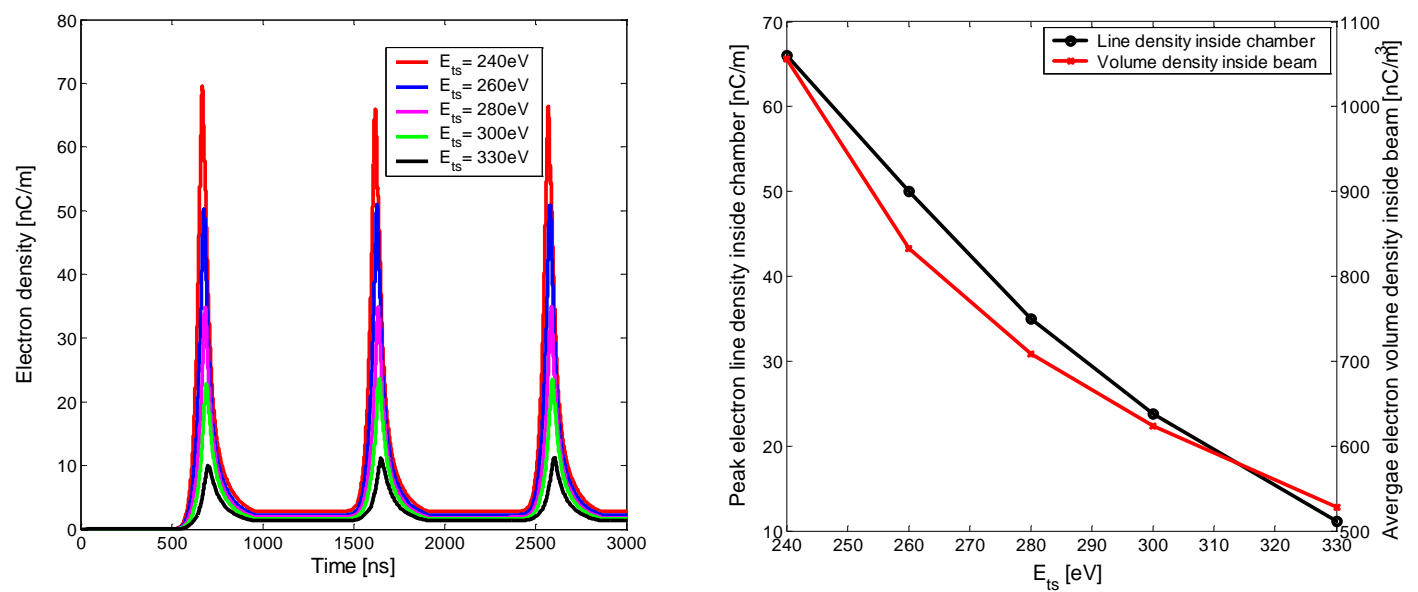

Figure 25 Effects of energy at peak SEY on electron build-up (left) and electron density (right).

\section{Electron by Ionization}

The number of electrons generated by the residual gas depends on the gas pressure and temperature. The yield usually is more than one order-of-magnitude less than the yield of electrons by proton loss when the vacuum is good. These electrons have low initial energy [36] and they cannot efficiently obtain energy from the beam when they are released at the end of bunch. Electrons have a maximum energy, $2.7 \mathrm{keV}$, for the SNS beam when they are created at the bunch's center with radial coordinate $a$. According to Eqs. (7) and (13), the possible maximum energy gain when an electron is released at the end of bunch is $130 \mathrm{eV}$. Figure 26 shows the buildup of the electron cloud for the SNS and PSR beams assuming that electrons are initially generated by ionization with a yield same as the yield by proton loss given by Table 1 . Note that there is no multipacting for both beams because of the low energy level of the electrons when they hit the chamber wall; the value is below $120 \mathrm{eV}$ for the SNS, and $60 \mathrm{eV}$ for PSR. The electron cloud accumulates 
slowly due to its long decay time at the bunch gap and the absence of multipacting. The electron-cloud density resulting from ionization is negligible compared with that due to the electrons generated by proton loss for both the SNS and PSR provided their vacuum pressure is satisfactory. When the vacuum is poor, the number of electrons generated by ionization will be notable and all them can be trapped inside beam and cause beam instability without strong multipacting. The electron cloud's density is roughly proportional to the chamber's vacuum pressure due to the lack of multipacting [37].
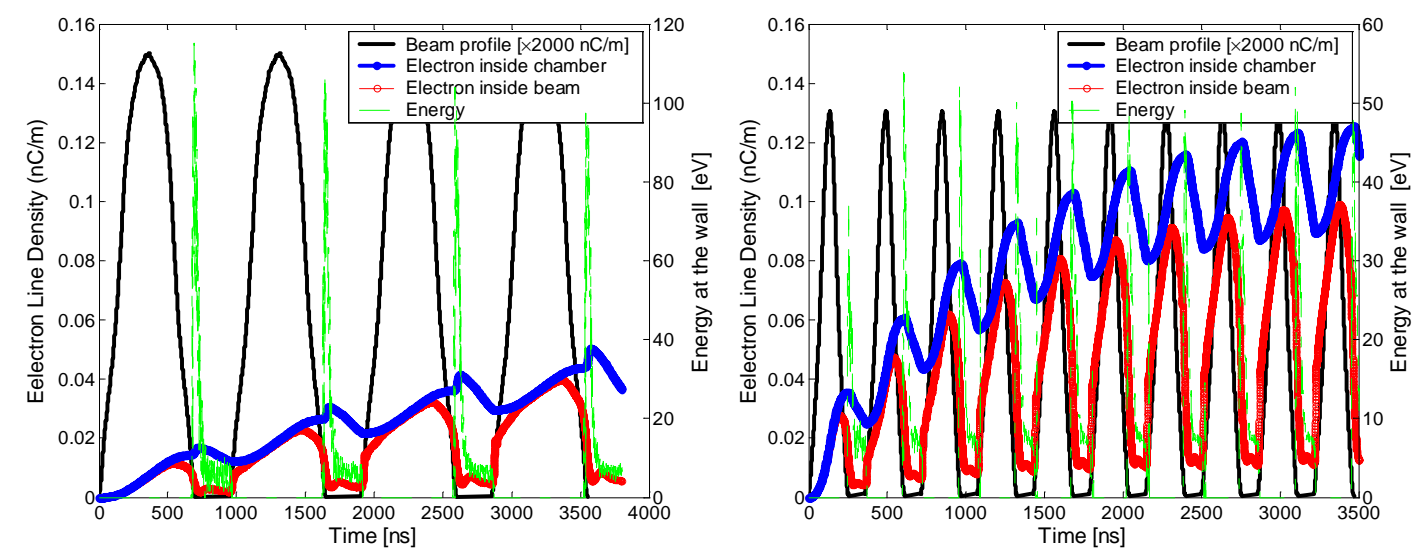

Figure 26 Electron cloud buildup due to ionization in the SNS's (left) and PSR's (right) drift region. The beam's profile was reduced by a factor of 2000 so that it could be clearly displayed together with electroncloud density

\section{Dipole Magnetic Field and Other Fields}

In dipole magnets, only electrons moving near the center of the horizontal chamber have enough energy at the wall's surface so that a multipacting cloud forms, as described by Eq. (39) and shown in Figure 12. Figure 27 shows the electron cloud buildup in the SNS's dipole magnet. The electron cloud is about two times smaller than that in drift region due to the limitation of the multipacting area in the dipole magnet. The simulated distribution of the electron cloud in a dipole magnet, shown in Figure 28, is consistent with the gain in electron energy gain (Figure 12). The electron cloud is trapped vertically by the beam's spacecharge force at the chamber's center during the beam's passage. Similar to the drift region, there is a strong multipacting at the bunch tail inside the dipole magnet. In the present proton machine, multipacting only can happen at the horizontal chamber's center because electron energy peaks there below a few hundreds $\mathrm{eV}$. It is less than $300 \mathrm{eV}$ in the SNS dipole magnet. However, in short bunch machines, for example the SPS and B-factories, the energy of an electron hitting the wall's surface at the center of the horizontal chamber could be more than thousands $\mathrm{eV}$ under normal operational parameters. This causes multipacting at two strips near the chamber's center [11,21].

Figure 29 shows the electron cloud's transverse distributions in a normal quadrupole magnet, a normal sextuple magnet, a solenoid, and the electric clearing electrode of the SNS ring. In quadrupole and sextuple magnets, very weak multipacting occurs around the middle of each magnetic pole because only those 
electrons moving along these field lines could receive enough energy by a mechanism similar to that inside a dipole magnet. The simulated electron cloud is more than two orders-of-magnitude smaller than in the drift region due to the low electron energy at the wall's surface. Quadrupole and sextuple magnet fields are mirror fields that may trap electrons via the mirror-field trap mechanism. However, trapping requires that the bunch length is shorter than the period of gyration [38]. Therefore, electrons emitted from the chamber's surface cannot be trapped in these magnets due to the long bunch length. The electron cloud distribution shown in Figure 29 implies that there is no mirror-field trap; the electron cloud stays closer to the mirror points of the field lines if mirror-field trapping happens. Compared with the electron cloud in the drifting region, the decay time of the electron cloud at the bunch gap in quadrupole and sextupole magnets is much longer due to the weak space-charge effect and the confinement of the electron's orbit by the magnetic fields. Figure 30 shows the electron cloud buildup and transverse distribution in the PSR quadrupole magnet. Similar to the SNS ring, the electron-cloud density in the PSR quadrupole is negligible and has long decay time due to the confinement of the electron's orbit by the magnetic fields.

A 30-Gauss weak solenoid can be invaluable in confining the electron cloud to the region near the wall and limiting the energy of electrons hitting the wall's surface to below the multipacting level. It can reduce the electron density inside chamber by a factor of a thousand. There is a non-electron circle region at the chamber's center with a radius more than the transverse beam's size. Macek's PSR experiment demonstrated that a 20-Gauss solenoid field reduces the electron signal by a factor of 50 [28]. We note that the solenoid field in that experiment was nonuniform, which has a weaker effect than a uniform one [39].

The effect of a clearing electrode is more complicated due to its disturbance of the electron's orbit. A weak voltage round $200 \mathrm{~V}$ can effectively suppress multipacting. On the other hand, a median clearing voltage, which is $2000 \mathrm{~V}$ in the SNS, can excite stronger multipacting than with zero clearing fields. We suggested how electron motion under a clearing field could explain the mechanism of action of the field [39].

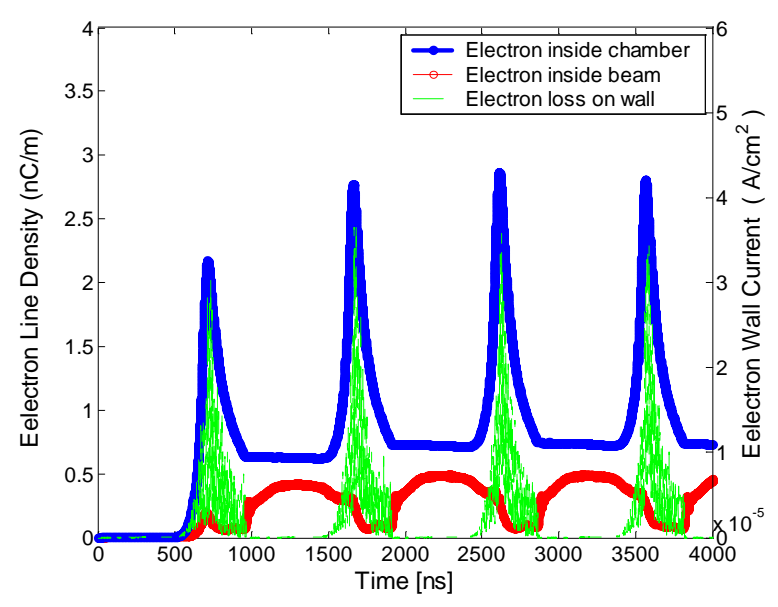

Figure 27 Electron cloud build-up in the SNS dipole 

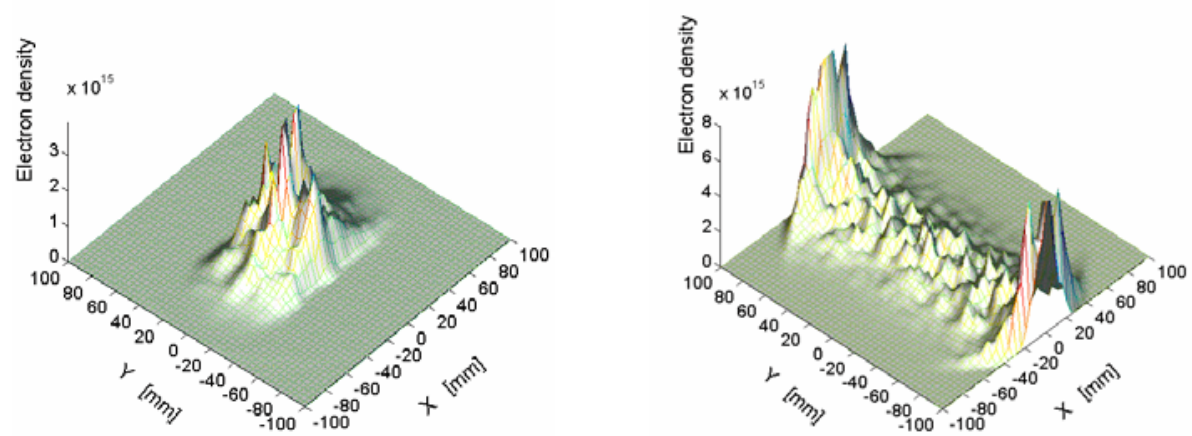

Figure 28 Electron cloud transverse distribution in the SNS dipole magnet at bunch center (left) and bunch tail (right)

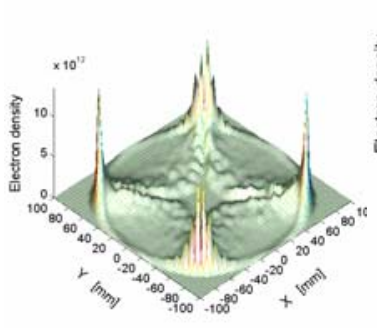

(a)

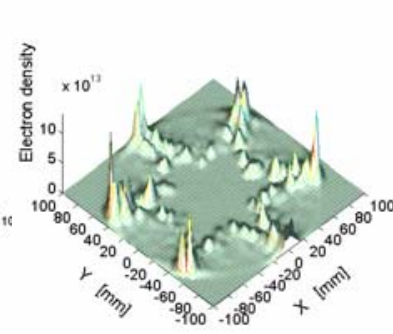

(b)

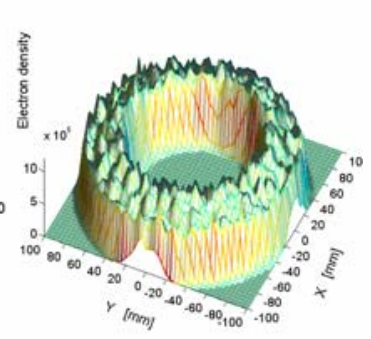

(c)

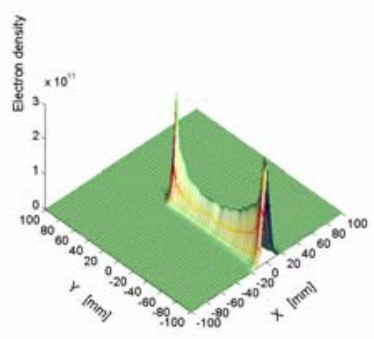

(d)

Figure 29 Electron transverse distributions in the SNS's quadrupole (a), sextuple (b), solenoid (c) magnets, and the clearing electrode (d)
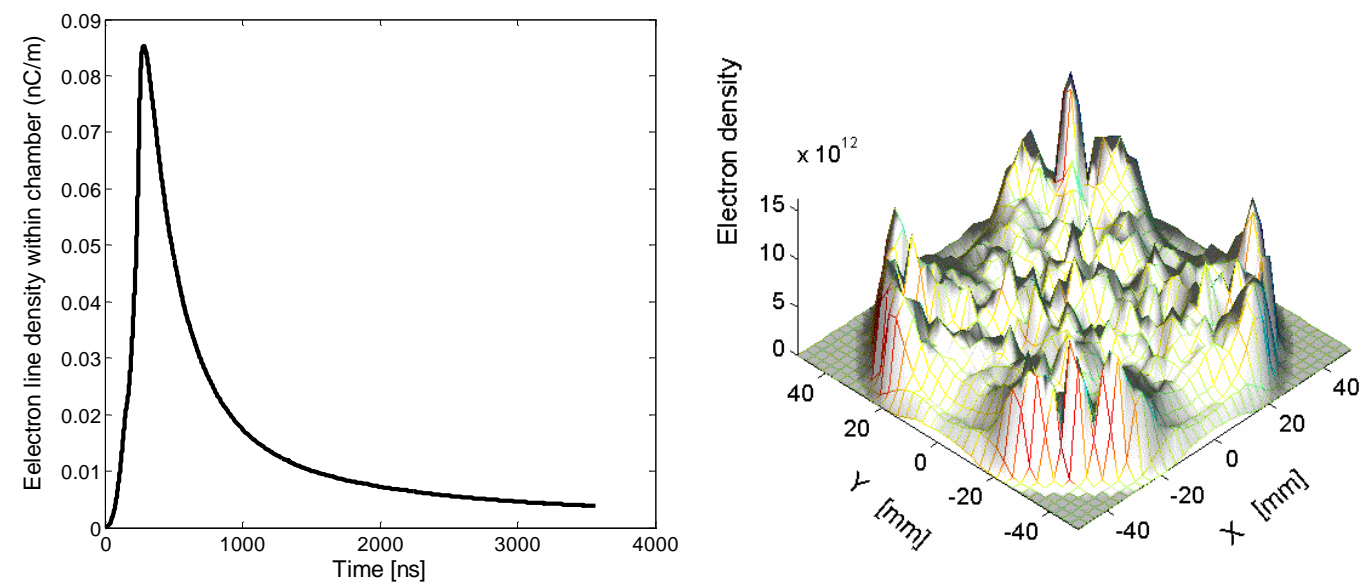

Figure 30 Electron cloud build-up (left), and transverse distribution at 225ns (right) in the PSR quadrupole magnet. 


\section{CONCLUSIONS}

We have studied in detail electron motion under the beam's space-charge field. The adiabatic invariant clearly describes the oscillation amplitude of the trapped electrons. Combining this data with the longitudinal beam's profile, we readily gained information about the trapped electrons, such as loss time and location. The electrons surviving from the last bunch gap cause proton beam instabilities due to their huge number and deep trapping inside beam during the beam's passage. However, these surviving electrons have weak effect on multipacting because they cannot receive enough energy from the beam. On the other hand, the straight drifting electrons can excite electron multipacting at the bunch tail. Our estimation of the gain in the straight drifting electron's energy when it hits the chamber wall is consistent with the numerical result. The energy gain of straight drifting electron clearly shows how and when multipacting occurs. According to the longitudinal beam profile factor, the gain in electron energy usually is bigger at the tail of the bunch. In consequence, the multipacting is stronger there. Our analysis quantitatively explains the mechanism of the "trailing edge multipactor".

Various factors related to the electron multipacting were investigated; the beam's longitudinal and transverse profiles, its intensity, the chamber's size, the bunch gap, and the SEY. Among them, multipacting is most sensitive to beam intensity. The electron density grows quickly with increases in the beam's intensity due to the combined effects of multipacting frequency and energy gain.

The longitudinal profile of the beam also plays a very important role on multipacting at the trailing edge. The longitudinal beam profile factor (Eq.40) can be used to directly estimate the beam profile's effect. According to our study, the bunch tail usually contributes to strong multipacting when the longitudinal beam profile factor is large. That can explain why cutting the bunch tail can effectively reduce multipacting. Thus, we can optimize the design of a real machine to reduce the beam profile factor. The energy spreader and corrector can significantly suppress the beam tail [40] and hence, reduce multipacting.

By contrast, the transverse beam profile has weak effects on electron multipacting. A Gaussian beam and a uniform cylindrical beam of the same RMS size exhibit the same electron-energy gain and electron cloud build-up. A beam with smaller transverse size contributes to stronger multipacting. Electron line density inside the chamber linearly decreases with transverse beam size while the electron volume density inside the beam decreases exponentially. Therefore, beam instability is more sensitive to transverse beam size,and a larger transverse size can weaken the electron-proton instabilities.

The bunch gap is important when the electron cloud's decay time is longer than the gap. In cases where the bunch gap is not long enough to clear the electron cloud, an electric clearing field can effectively do so. The electron cloud surviving from the bunch gap between subsequent beam bunches, and hence, beam instabilities, also is sensitive to the beam in gap. Multistage beam cleaning that includes multi-step chopping at low energies and beam-in-gap sweeping with collimator collection at the top energy ensures a clean gap.

The secondary emission parameters directly affect electron multipacting. The electron cloud within the chamber shows a roughly linearly increase with the increase of peak SEY in the SNS ring. However, the 
electron cloud within the beam is saturated at high SEY due to the strong space-charge force at the bunch gap. Except for the peak SEY, energy at the peak SEY also has a very important effect on a long bunch because the maximum gain in energy is close to the energy at the peak SEY. Increasing the energy at peak SEY can significantly reduce multipacting and hence, electron-proton instabilities.

The size of the chamber has both advantages and disadvantages on electron multipacting. Larger chambers entail a larger electron-energy gain and lower multipacting frequency.

With a good vacuum, electrons generated by ionization have a weak effect on the beam's stability due to the absence of multipacting. When the vacuum is poor, these electrons will excite strong electron-proton instabilities because they can be trapped deep inside the beam during its passage.

Multipacting happens at the chamber's center in a dipole magnet. The electron density in dipole magnet is reduced to one-third of the electron density in drift region due to the limitation of multipacting area in dipole magnet. There is a weak multipacting in quadrupole and sextuple magnets where the electron density is two orders-of-magnitude lower than in the drift region. There is no mirror-field trapping in the quadrupole magnet due to the long bunch length. A weak solenoid field up to 30-Gauss can confine all electrons near the wall surface and reduce the electron density with a factor one thousand in the drift region. A clearing electrode also works, but has a complicated effect that depends on the clearing field.

\section{ACKNOWLEDGMENTS}

We thank Professor A. Chao, A. Fedotov, P. He, Y.Y. Lee, D. Raparia, T. S.Wang, A. Browman, S.Y. Zhang, and F. Zimmermann for helpful discussions.

\section{REFERENCE}

[1] G.I. Budker, G.I. Dimov, V.G. Dudnikov, Proceedings of the International Symposium on Electron and Positron Storage Rings, VIII-6-1, Saclay, 1966.

[2] H.G. Hereward, CERN Report No. 71-15, 1971

[3] E. Keil and B. Zotter, CERN Report No. CERN-ISR-TH/71-58, 1971

[4] R. J. Macek, A. Browman, D. Fitzgerald, R.C. McCrady, F. E. Merrill, M. A. Plum, T. Spickermann, T. S. Wang, K. C. Harkay, R. Kustom, R. A. Rosenberg, J. E. Griffin, K.Y. Ng, and D. Wildman, Proceedings of the Particle Accelerator Conference, Chicago, 2001 (IEEE, Piscataway, NJ, 2001), p. 688.

[5] M. Blaskiewicz, Workshop on Instabilities of High Intensity Hadron Beams in Rings, edited by T. Roser and S.Y. Zhang, AIP Conf. Proc. No. 496 (AIP, New York, 1999), p. 321.

[6] M.Izawa, Y. Sato, and T. Toyamasu, Phys. Rev. Lett. 74, 5044-5047,1995

[7] K. Ohmi, Phys. Rev. Lett. 75, 1526-1529, 1995

[8] H. Fukuma, Mini-Workshop on Electron Cloud Simulation for Proton and Positron Beams, Proceedings ECLOUD'02, CERN-2002-001, Edited by G. Rumolo and F. Zimmerman, 2002, pp.1-10. 
[9] Sam Heifets, Proceedings of 8th Advanced Beam Dynamics Mini-workshop on Two-stream Instabilities in Particle Accelerators and Storage Rings, Santa Fe, New Mexico, USA, 2000. URL: http://www.aps.anl.gov/conferences/icfa/two-stream.html.

[10] Z.Y. Guo, H. Huang, S.P. Li, D.K. Liu, L. Ma, Q. Qin, L.F. Wang, J.Q. Wang, S.H. Wang, C. Zhang, F. Zhou, Y.H. Chin, H. Fukuma, S. Hiramatsu, M. Izawa, T. Kasuga, E. Kikutani, Y. Kobayashi, S. Kurokawa, K. Ohmi, Y. Sato, Y. Suetsugu, M. Tobiyama, K. Yokoya, X.L. Zhang, KEK-PREPRINT-98-23, 1998; also Proceedings of 1st Asian Particle Accelerator Conference, Tsukuba, Japan, 1998, pp 432-434. Edited by Y.H. Chin, M. Kihara, H. Kobayashi, N. Akasaka, K. Nigorikawa, Tobiyama.

[11] J.M. Jiménez, G. Arduini, P. Collier, G. Ferioli, B. Henrist, N. Hilleret, L. Jensen, K. Weiss, F. Zimmermann, "Electron Cloud with LHC-type beams in the SPS : a review of three years of measurements", LHC-Project-Report-632 (2003) .

[12] K. Cornlis, Mini-Workshop on Electron Cloud Simulation for Proton and Positron Beams, Proceedings ECLOUD’02, CERN-2002-001, Edited by G. Rumolo and F. Zimmerman, 2002, pp.11-16.

[13] O. Grőbner, Proceedings of the 10th International Accelerator Conference, Protvino, Russia, 1977 (Institute of High Energy Physics, Protvino, 1977), p. 277.

[14] O. Gróbner, Proceedings of the 17th IEEE Particle Accelerator Conference (PAC 97), Vancouver, Canada (IEEE, Piscataway, NJ, 1998), p. 3589.

[15]R. Macek, "Sources of electrons for stable beams in PSR”, PSR Technical Note, PSR-00-10; also V. Danilov, A. Aleksandrov, J. Galambos, D. Jeon, J. Holmes, D. Olsen, "Multipacting on the Trailing Edge of Proton Beam Bunches in PSR and SNS”, Workshop on Instabilities of High Intensity Hadron Beams in Rings, edited by T. Roser and S.Y. Zhang, AIP Conf. Proc. No. 496 (AIP, New York, 1999), p. 315.

[16] M. Blaskiewicz, M. A. Furman and M. Pivi, Physics Review Special Topics-Accelerators and Beams, Vol.6, 014203(2003).

[17]M.T.F. Pivi and M.A. Furman, Physics Review Special Topics-Accelerators and Beams, Vol.6, 034201(2003).

[18] K. Ohmi, T. tama and C. Ohmori, Physics Review Special Topics-Accelerators and Beams, Vol.5, 114402(2002).

[19] J. Wei and R. J. Macek, Mini-Workshop on Electron Cloud Simulation for Proton and Positron Beams, Proceedings ECLOUD’02, CERN-2002-001, Edited by G. Rumolo and F. Zimmerman, 2002, pp.29-40.

[20] M.A. Furman and M.T.F. Pivi, LBNL-52872 NOTE 516, 2003, (unpublished)

[21]L. F. Wang, H. Fukuma, K. Ohmi, S. Kurokawa and K. Oide, F. Zimmermann, Physics Review Special Topics-Accelerators and Beams, Vol. 5, 124402 (2002)

[22] M.A. Furman and M.T.F. Pivi, Physics Review Special Topics-Accelerators and Beams, Vol.5, 124404(2002).

[23] R. Cimino, "The electron cloud problem and potential remedies". ICFA Mini-workshop-Working group on High Luminosity e+e- Colliders, 10-13 September 2003, Alghero (SS), Italy, http://www.lnf.infn.it/conference/d2/gener.htm 
[24] A.V. Fedotov, J. Wei, R.L. Gluckstern, Proceedings of the 2001 Particle Accelerator Conference, Chicago, Illinois, 2001, p. 2851.

[25] R. J. Macek, LANL PSR TECH NOTE, PSR-98-04, 1998

[26] D. Neuffer, E. Colton, D. Fitzgerald, T. Hardek, R. Hutson, R. Macek, M. Plum, Henry A. Thiessen, T.S. Wang, Nucl. Instrum. Meth. A 321, 1992, p.1-12.

[27]Lehnert, B., Progress in Nuclear Energy, edited by J.L. Tuck, Series XI, Vol. II, Pergamon Press, 1962

[28]R. J. Macek, Mini-Workshop on Electron Cloud Simulation for Proton and Positron Beams, Proceedings ECLOUD’02, CERN-2002-001, Edited by G. Rumolo and F. Zimmerman, 2002, pp. 259268

[29] M. Plum, D. Johnson, R. Macek, F. Merrill, B. Prichard, PSR TECH NOTE, PSR-97-19(1997)

[30] K. Ohmi and F. Zimmermann, Phys. Rev. Lett. 85, p.3821-3823 (2000).

[31] The data collection was done by detector ED42Y in LANL PSR, July 2, 2002, and is documented in Log Book 95, p6-7. Courtesy R. Macek.

[32] B. Houssais, Thesis, Univ. de Rennes, France, 1967.

[33]A. Browman, Proceedings of 8th advanced beam dynamics mini-workshop on two-stream instabilities in particle accelerators and storage rings, Santa Fe, New Mexico, USA, 2000. URL: http://www.aps.anl.gov/conferences/icfa/two-stream.html.

[34] R. Macek, M. Borden, A. Browman, D. Fitzgerald, T.S. Wang, T. Zaugg, K. Harkay, R.A. Rosenberg, Proceedings of the 2003 Particle Accelerator Conference (PAC 03), Portland, Oregon, USA (IEEE, Portland, Oregon, 2003), ROAB003, 2003.

[35] The data was collected in Oct. 7, 2001 for a sweeping detector of PSR, and is documented in Log Book 98, p132-3. Courtesy R. Macek and A. Browman

[36] Lapique F. and Piuz F., Nucl. Instrum. Methods Phys. Res. , 175 (1980) , p.297-318

[37] R. Macek, The 13th ICFA Beam Dynamics Mini-Workshop, Brookhaven National Laboratory, Upton, NY, December, 2003(Unpublished). http://www.c-ad.bnl.gov/icfa/

[38] L. F. Wang, H. Fukuma, S. Kurokawa, K. Oide, Physic Review E, 66, 036502(2002).

[39]L. F. Wang, D. Raparia, J. Wei and S.Y. Zhang, accepted by Physics Review Special TopicsAccelerators and Beams.

[40] Jie Wei, Reviews of Modern Physics, Volume 75(2003), p.1383-1432. 